\title{
Copper-Catalyzed Three-Component Tandem Cyclization for One-Pot Synthesis of 1,4-Benzothiazines
}

\author{
Jing-Jing Chu, Bo-Lun Hu, Zhi-Yong Liao and Xing-Guo Zhang* \\ College of Chemistry and Materials Engineering, Wenzhou University, Wenzhou \\ 325035, China
}

$\underline{\text { zxg@,wzu.edu.cn }}$

Supporting Information

contents

NMR Spectra for All Compounds.

S2-S57 


\section{${ }^{1}$ H NMR of 3-phenyl-4H-benzo[b][1,4]thiazine-4-carbonitrile (3a)}
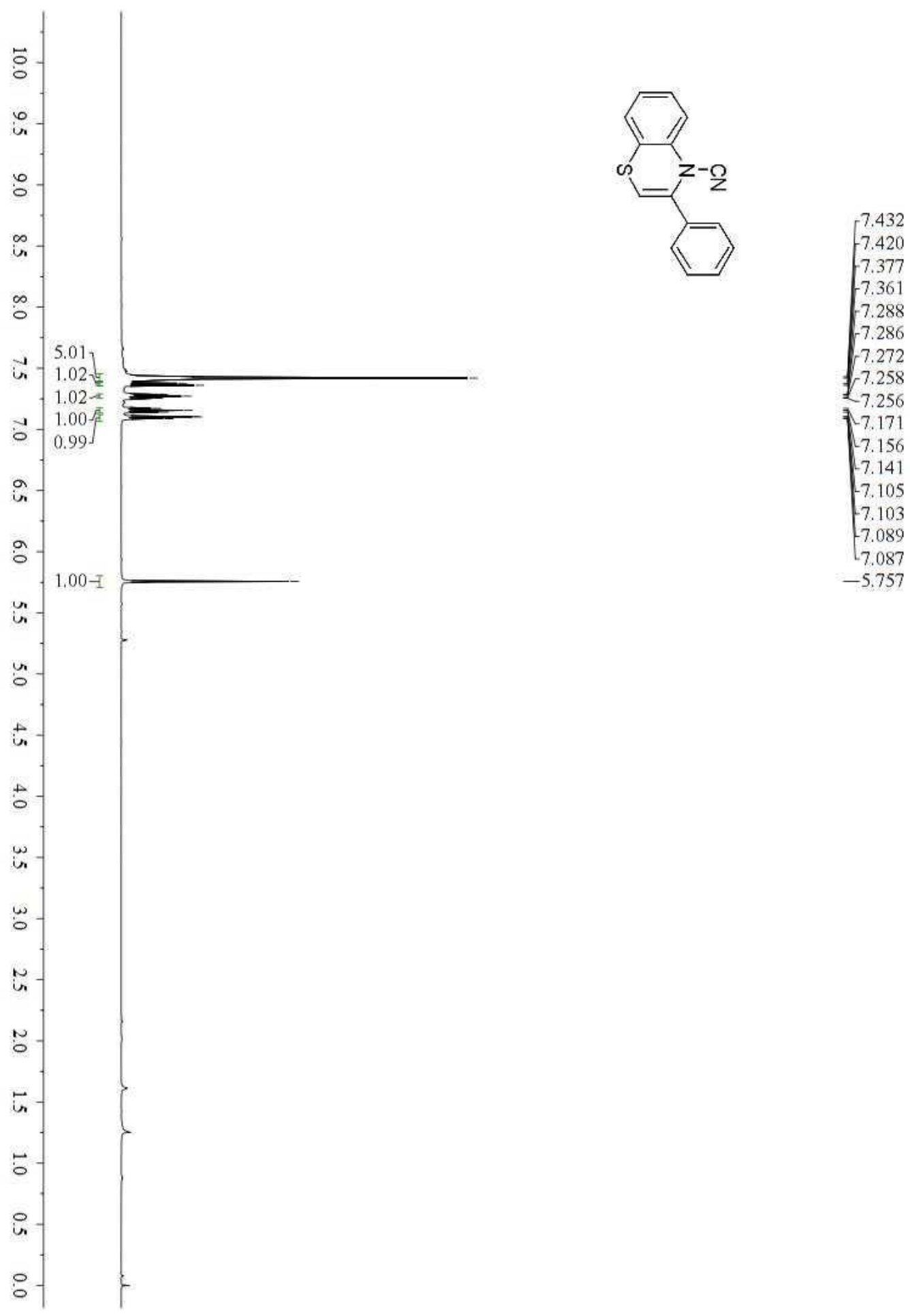


\section{${ }^{13} \mathrm{C}$ NMR of 3-phenyl-4H-benzo[b][1,4] thiazine-4-carbonitrile (3a)}
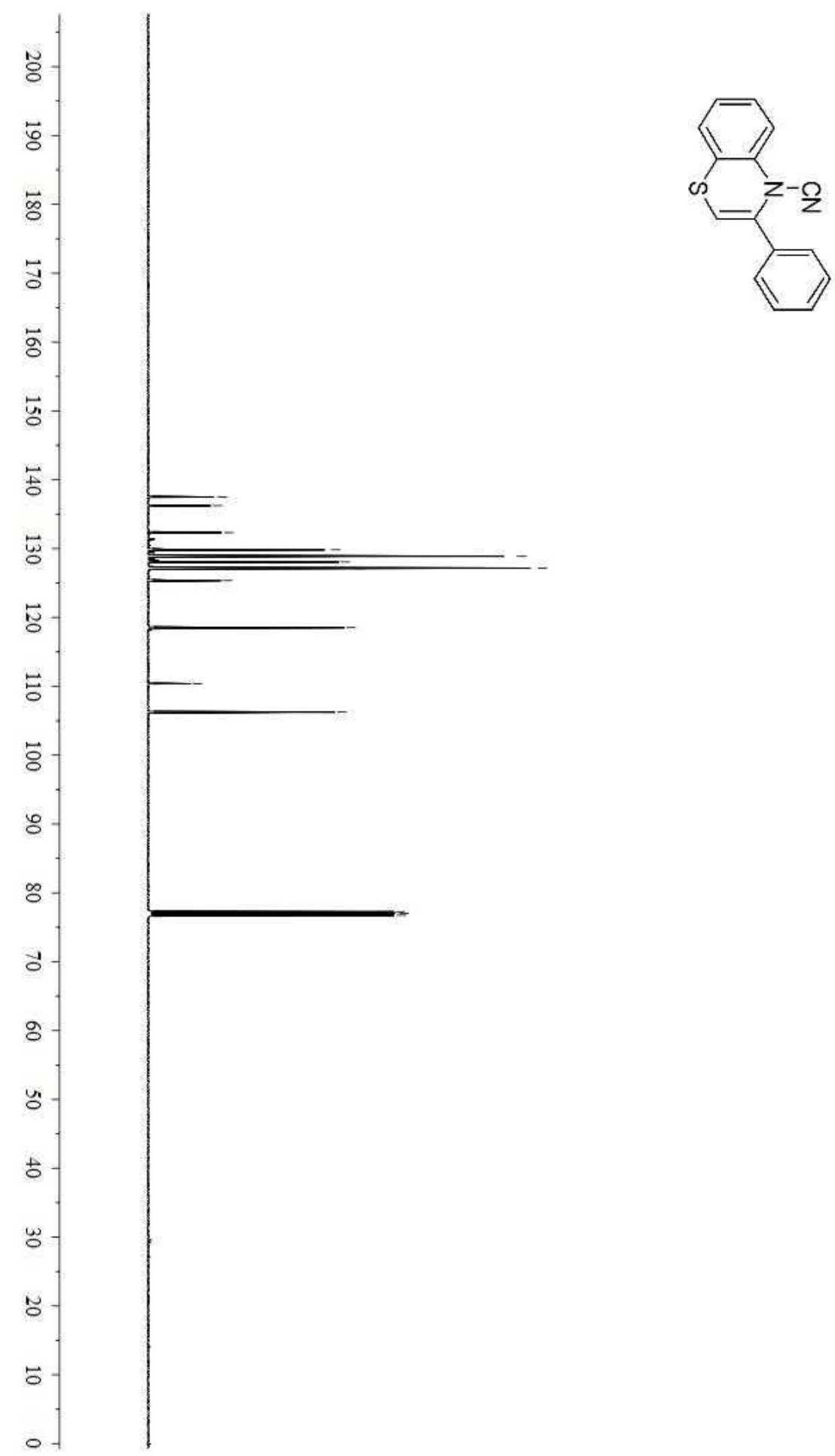
${ }^{1}$ H NMR of 3-(3-fluorophenyl)-4H-benzo[b][1,4]thiazine-4-carbonitrile (3b)
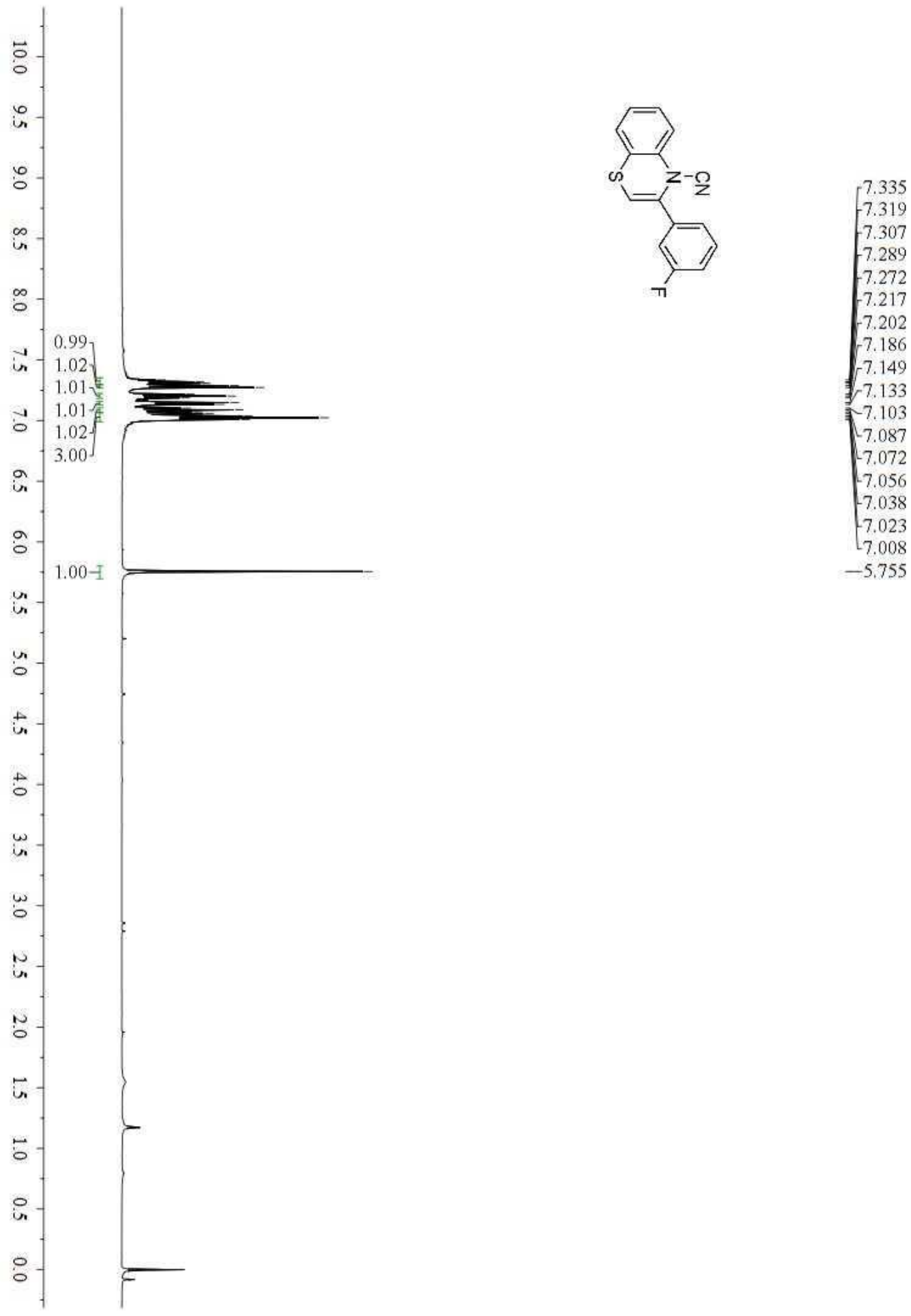
${ }^{13}$ C NMR of 3-(3-fluorophenyl)-4H-benzo[b][1,4]thiazine-4-carbonitrile (3b)
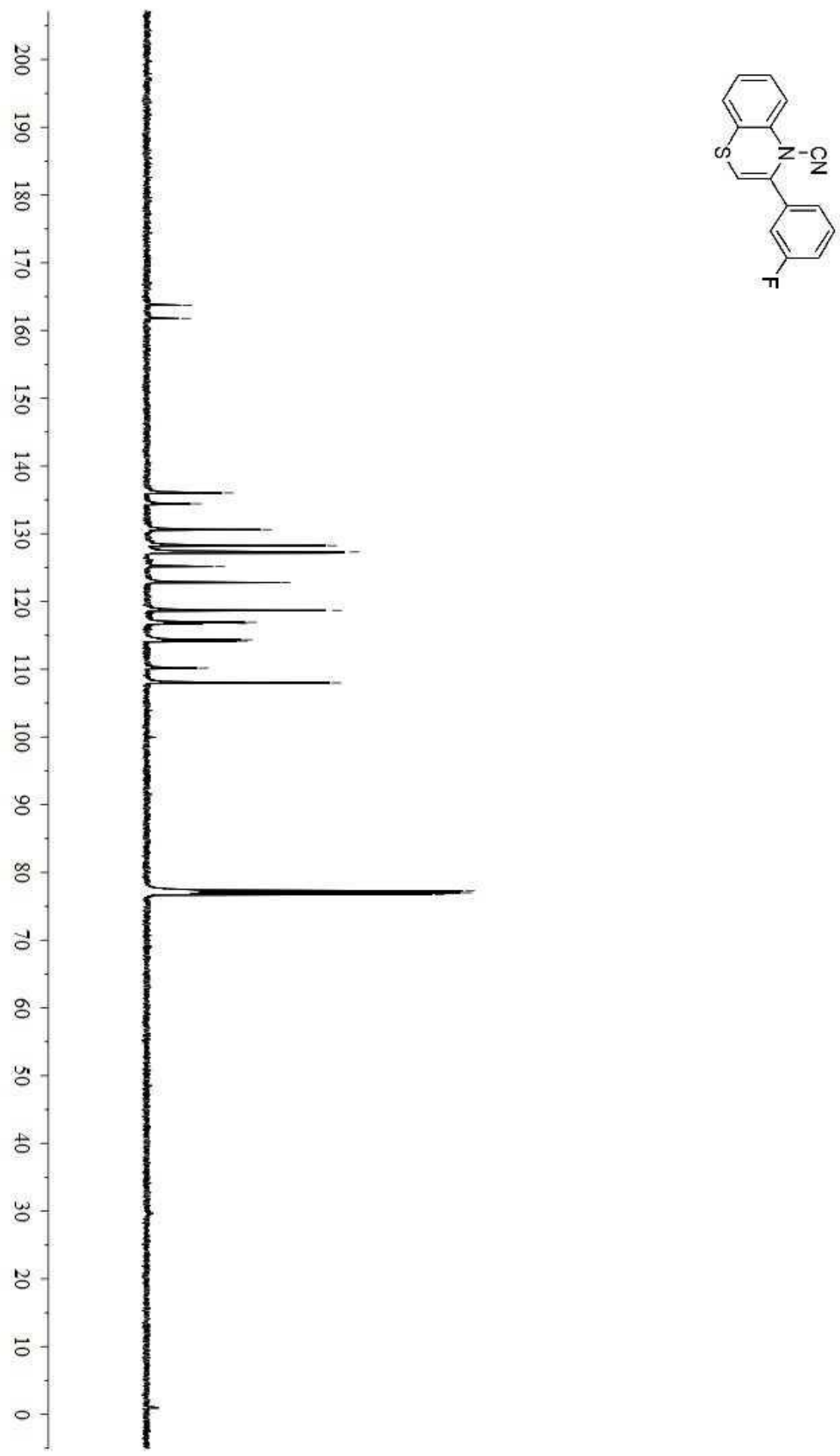

$-163.747$

$-161.775$

136.027

$-134.416$

$-134.354$

130.602

$-130.535$

128.233

$-127.296$

$\gamma_{127.135}$

$-125.155$

$-122.784$

$-118.676$

$-116.929$

$-116.761$

$-114.337$

$-114.153$

$-110.165$

107.989

77.255

$-77.000$

$-76.747$ 
${ }^{1}$ H NMR of 3-(4-fluorophenyl)-4H-benzo[b] [1,4]thiazine-4-carbonitrile (3c)

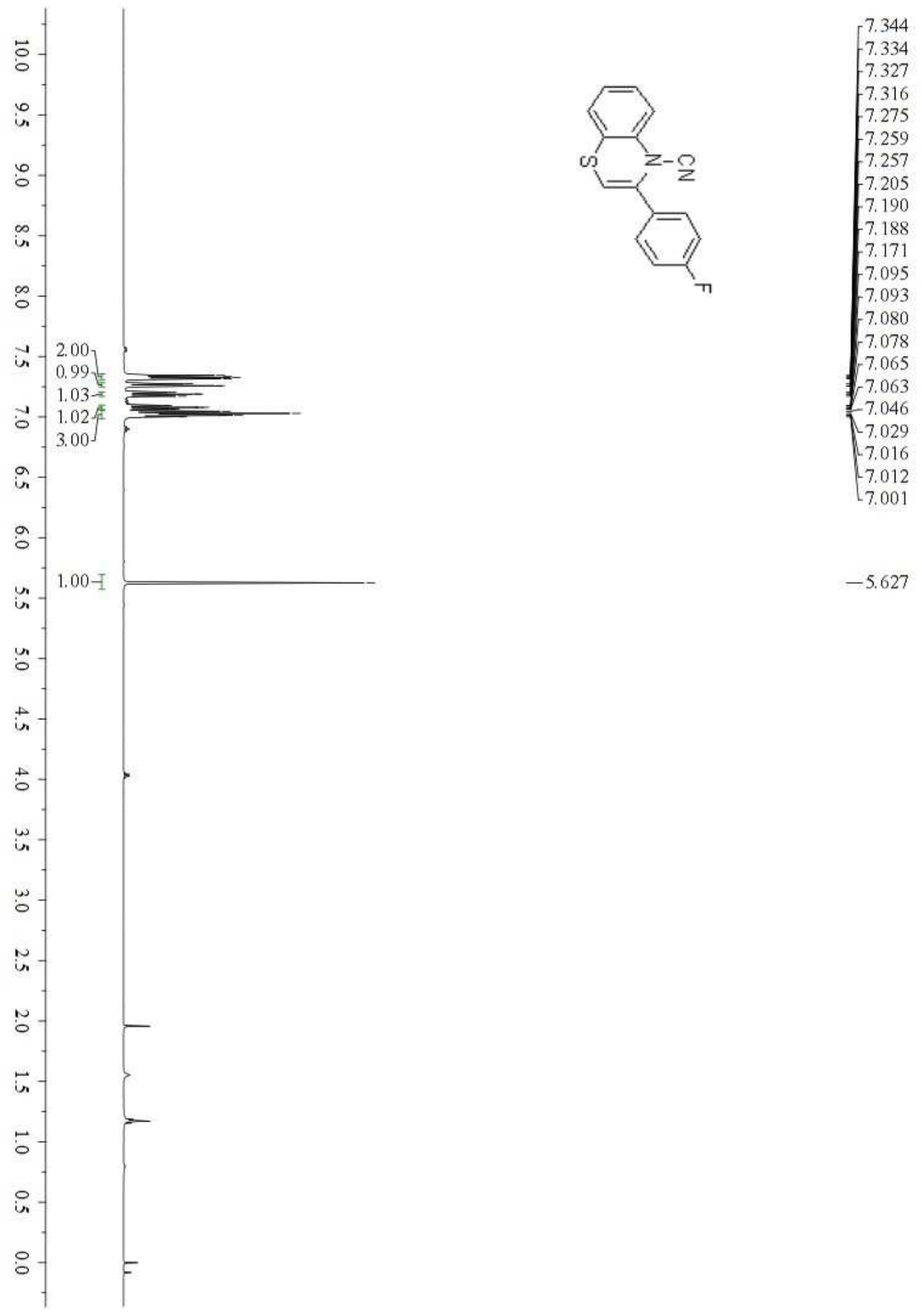


${ }^{13}$ C NMR of 3-(4-fluorophenyl)-4H-benzo[b][1,4]thiazine-4-carbonitrile (3c)
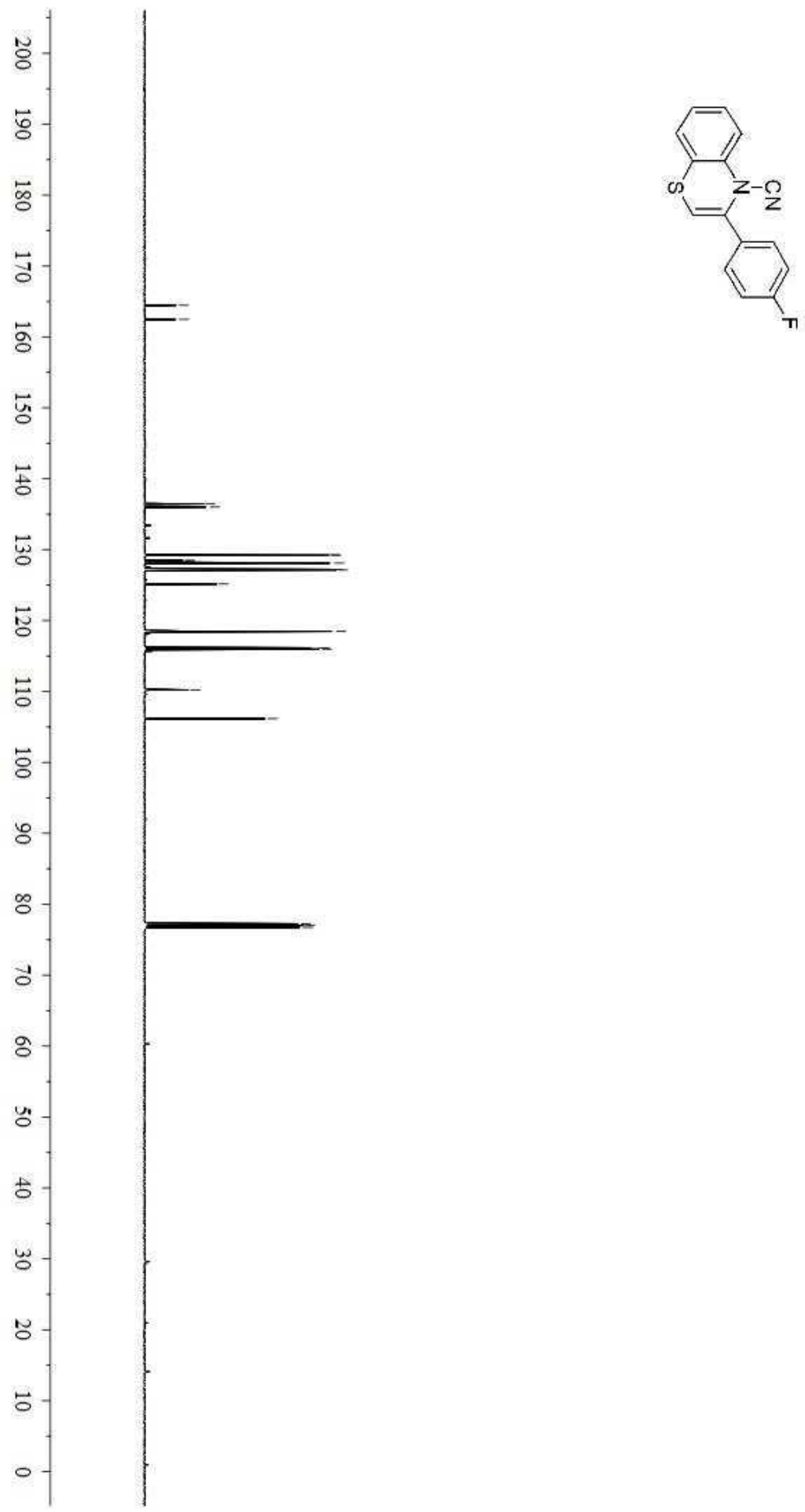

$-164.459$

$-162.465$

$\left[\begin{array}{r}136.456 \\ 136.023\end{array}\right.$

129.283

129.215

$-128.467$

$-128.440$

$-128.109$

$-127.221$

127.135

125.164

$-118.483$

$-116.155$

$-115.980$

$-110.221$

$-106.177$

77.255

$-77.000$

$-76.746$ 
${ }^{1}$ H NMR of 3-(2-fluorophenyl)-4H-benzo[b][1,4]thiazine-4-carbonitrile (3d)
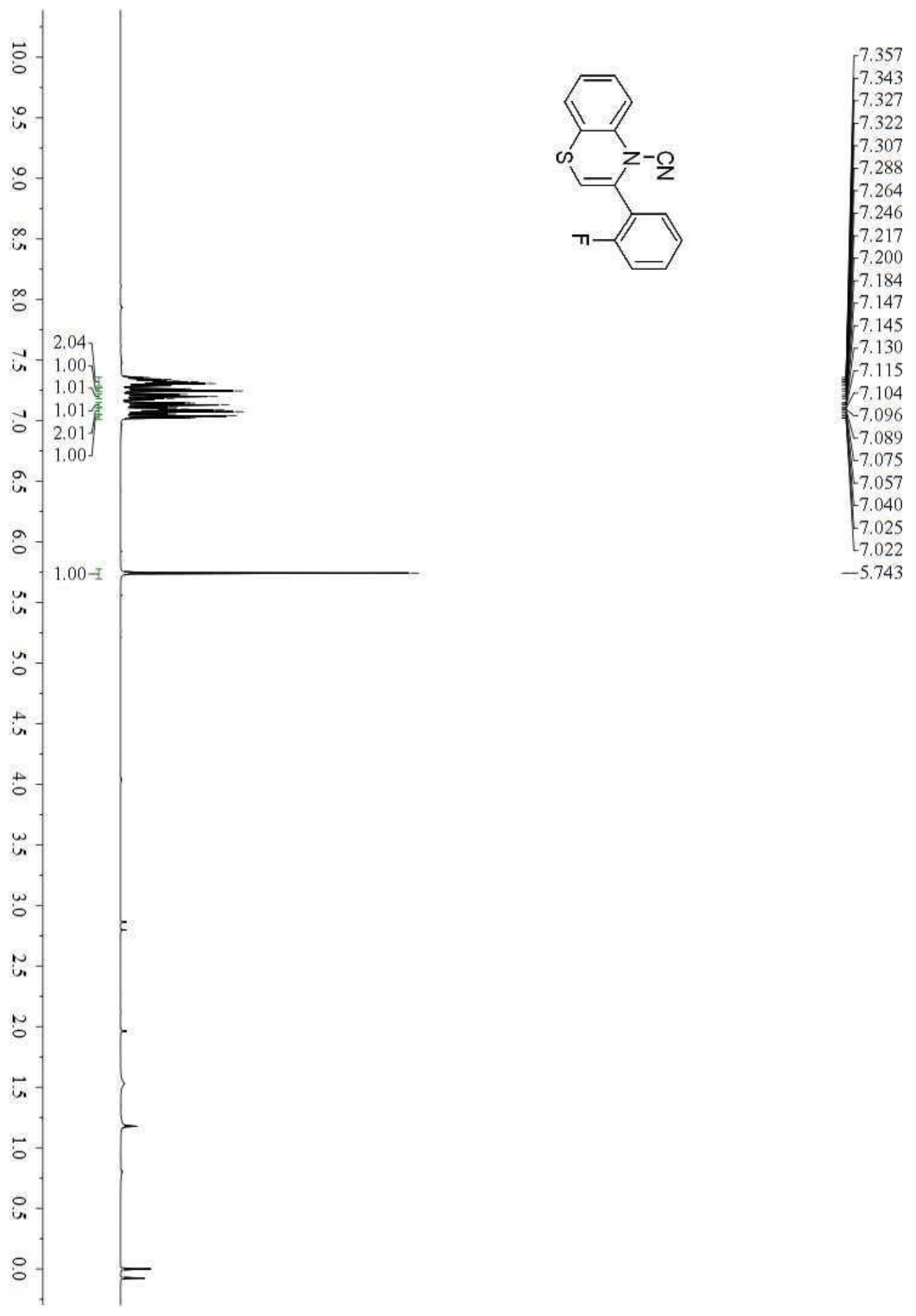
${ }^{13}$ C NMR of 3-(2-fluorophenyl)-4H-benzo[b][1,4]thiazine-4-carbonitrile (3d)
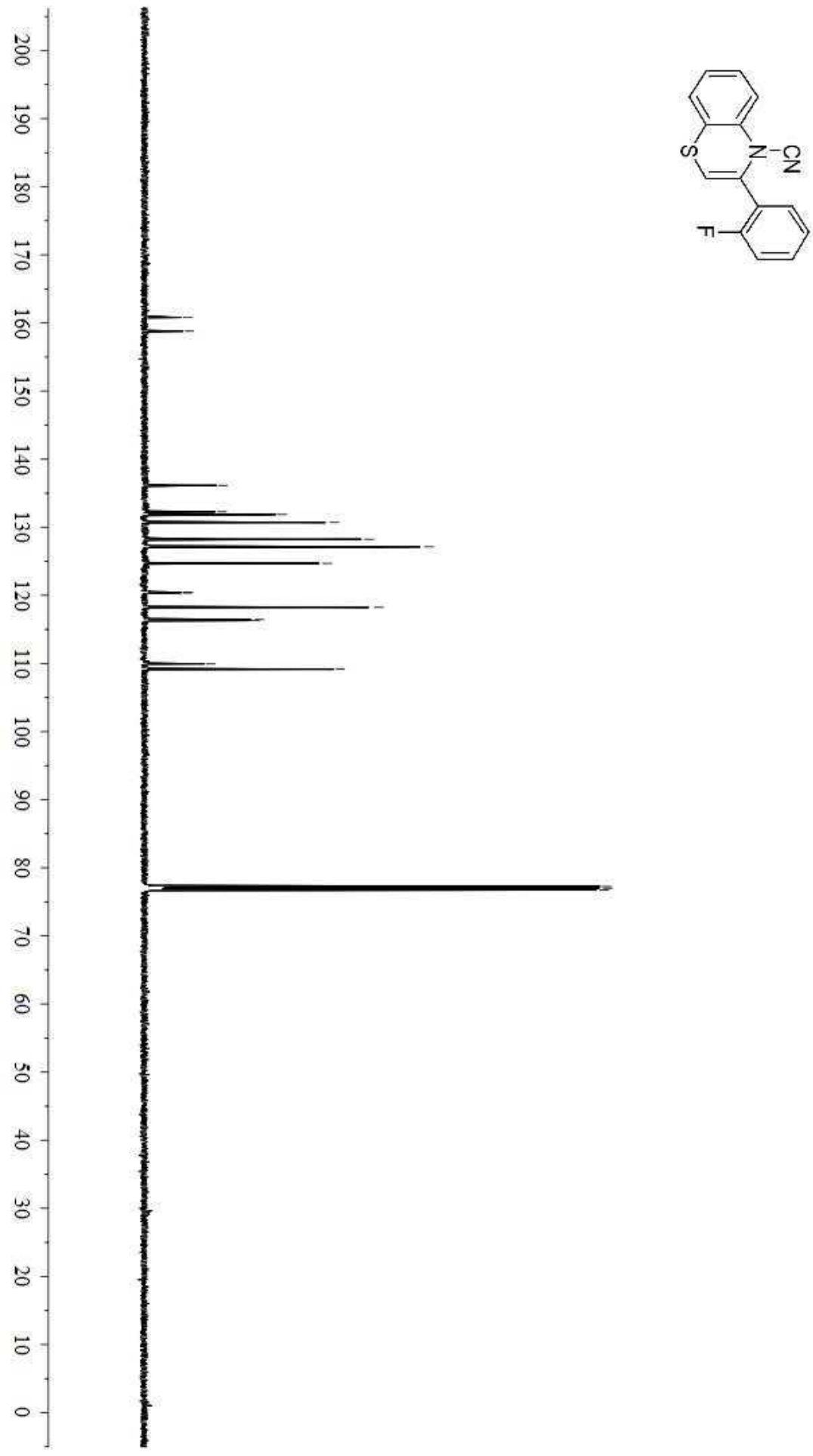

$-160.793$

$-158.795$

136.143

$-132.274$

$-131.874$

$-131.807$

130.706

128.233

- -127.135

- 127.082

$-124.704$

124.676

124.645

$-120.457$

$-120.352$

$-118.236$

$-116.452$

$-116.282$

$-109.942$

$-109.157$

77.255

$-77.000$

$-76.746$ 
${ }^{1}$ H NMR of 3-(4-chlorophenyl)-4H-benzo[b][1,4]thiazine-4-carbonitrile (3e)
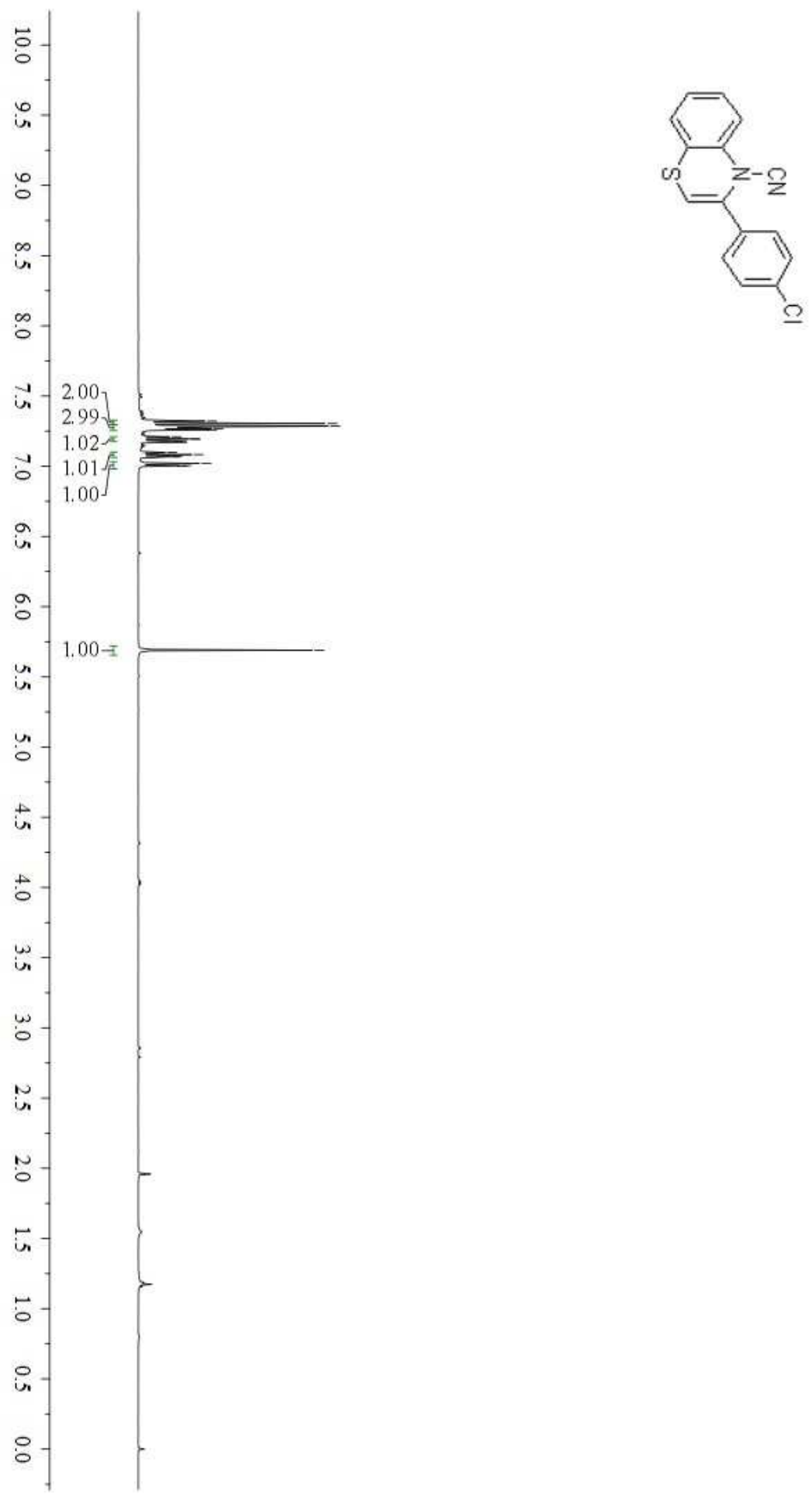

$$
\left\{\begin{array}{r}
7.321 \\
7.304 \\
7.285 \\
7.268 \\
7.259 \\
7.210 \\
7.195 \\
7.193 \\
7.177 \\
7.099 \\
7.083 \\
7.068 \\
7.066 \\
7.020 \\
7.005 \\
7.002
\end{array}\right.
$$


${ }^{13}$ C NMR of 3-(4-chlorophenyl)-4H-benzo[b][1,4]thiazine-4-carbonitrile (3e)
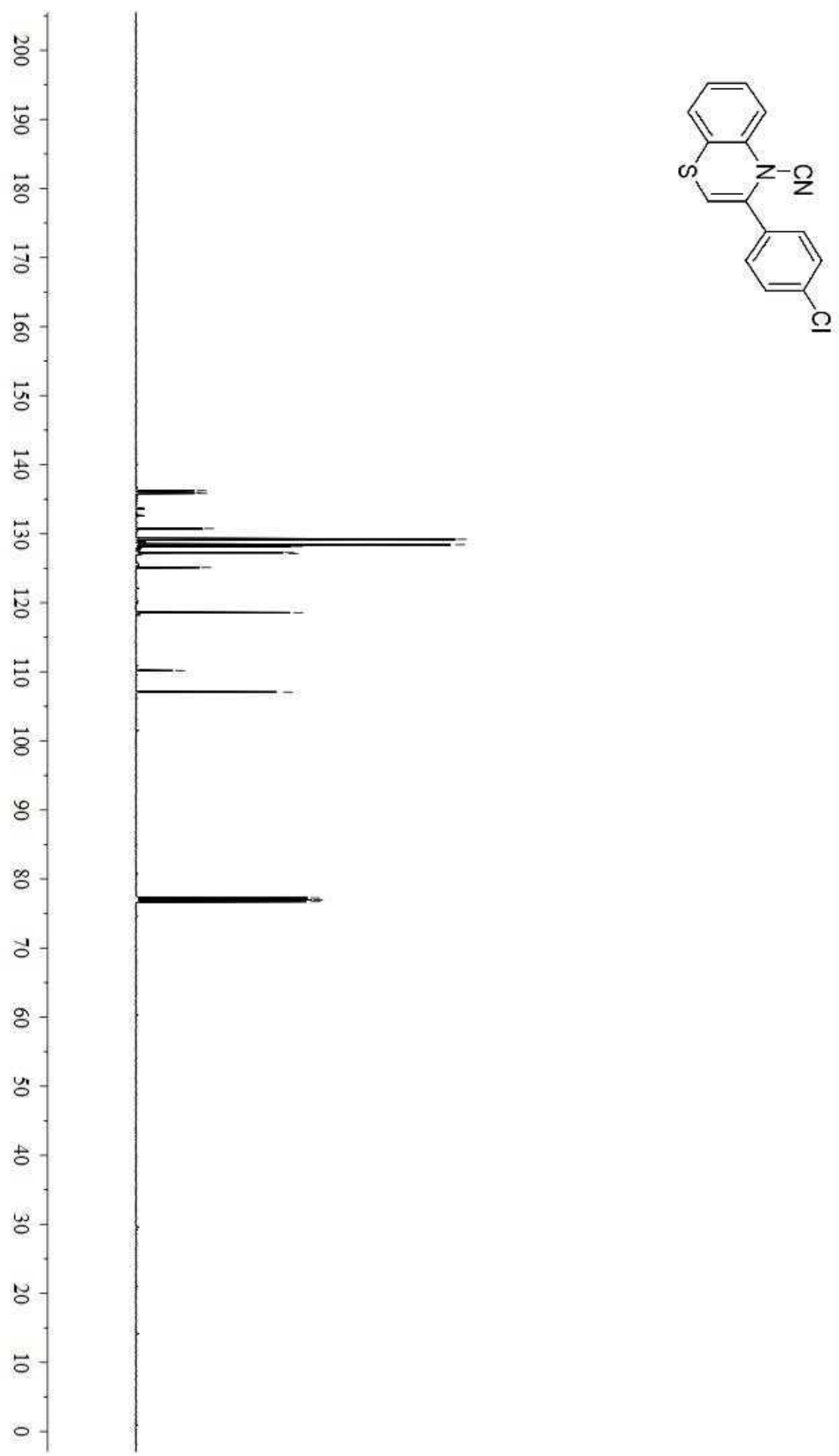

136.238

$-135.951$

$-135.848$

130.753

129.193

$-128.413$

$-128.175$

127.265

127.137

125.116

$-118.565$

$-110.182$

$-107.073$

$\left\{\begin{array}{r}77.254 \\ -77.000\end{array}\right.$

76.746 
${ }^{1}$ H NMR of 3-(3-chlorophenyl)-4H-benzo[b][1,4]thiazine-4-carbonitrile (3f)
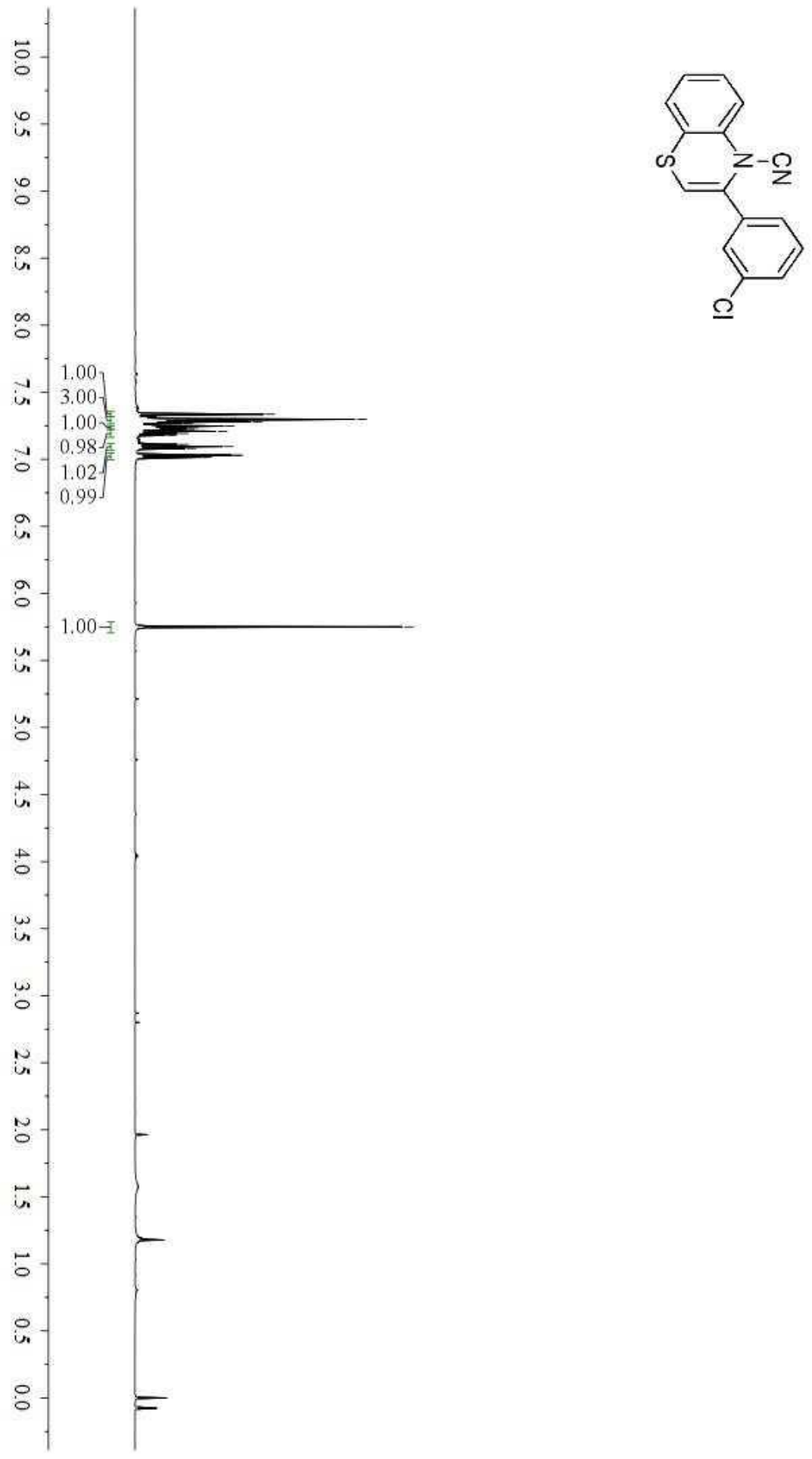

$\left[\begin{array}{r}7.336 \\ 7.298 \\ 7.280 \\ 7.269 \\ 7.251 \\ 7.248 \\ 7.234 \\ 7.223 \\ 7.209 \\ 7.192 \\ 7.178 \\ 7.111 \\ 7.096 \\ 7.081 \\ 7.031 \\ 7.029 \\ 7.016 \\ 7.014 \\ -5.750\end{array}\right.$ 
${ }^{13}$ C NMR of 3-(3-chlorophenyl)-4H-benzo[b] $[1,4]$ thiazine-4-carbonitrile (3f)

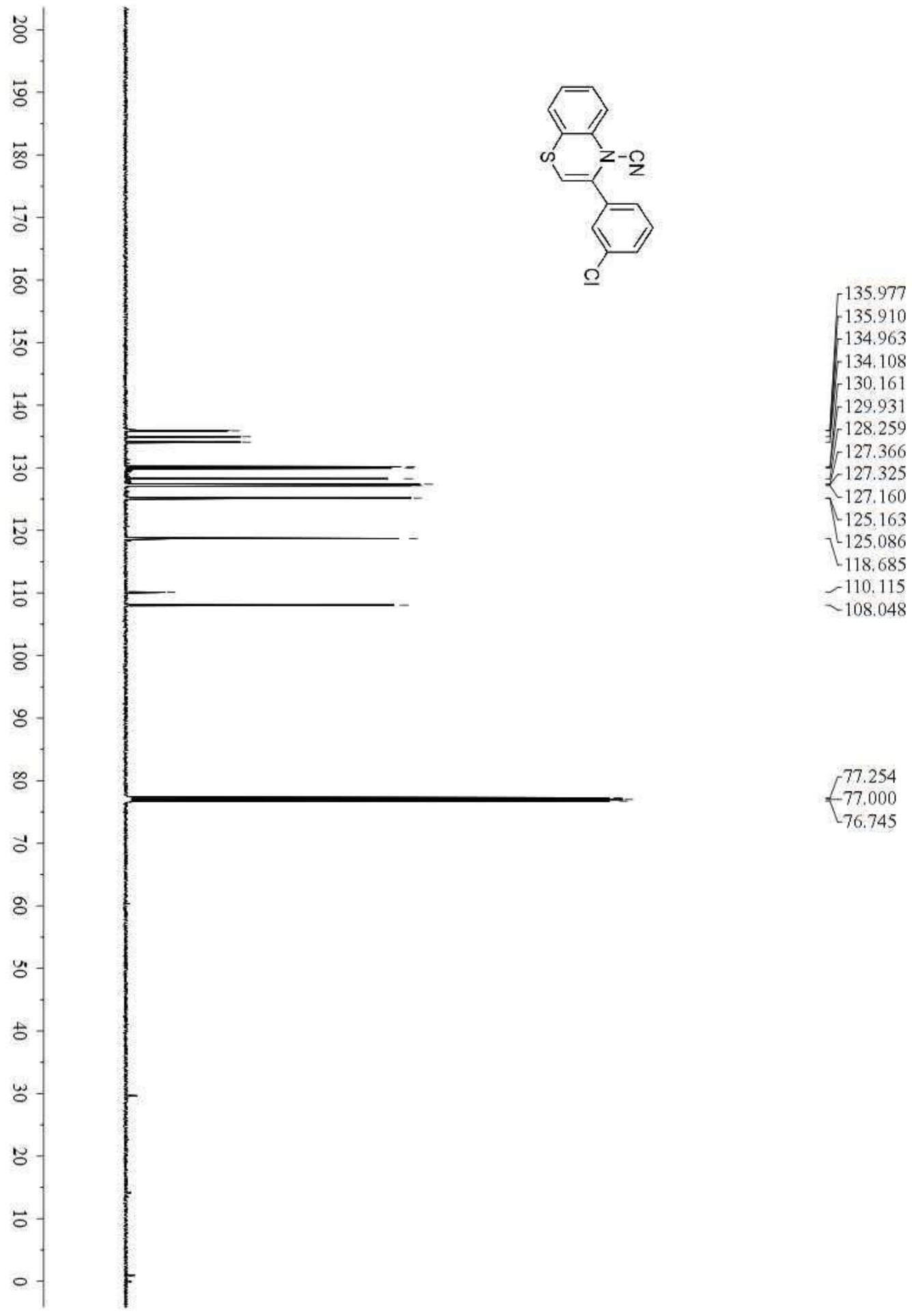


${ }^{1} \mathrm{H}$ NMR of 3-(4-bromophenyl)-4H-benzo[b][1,4]thiazine-4-carbonitrile (3g)
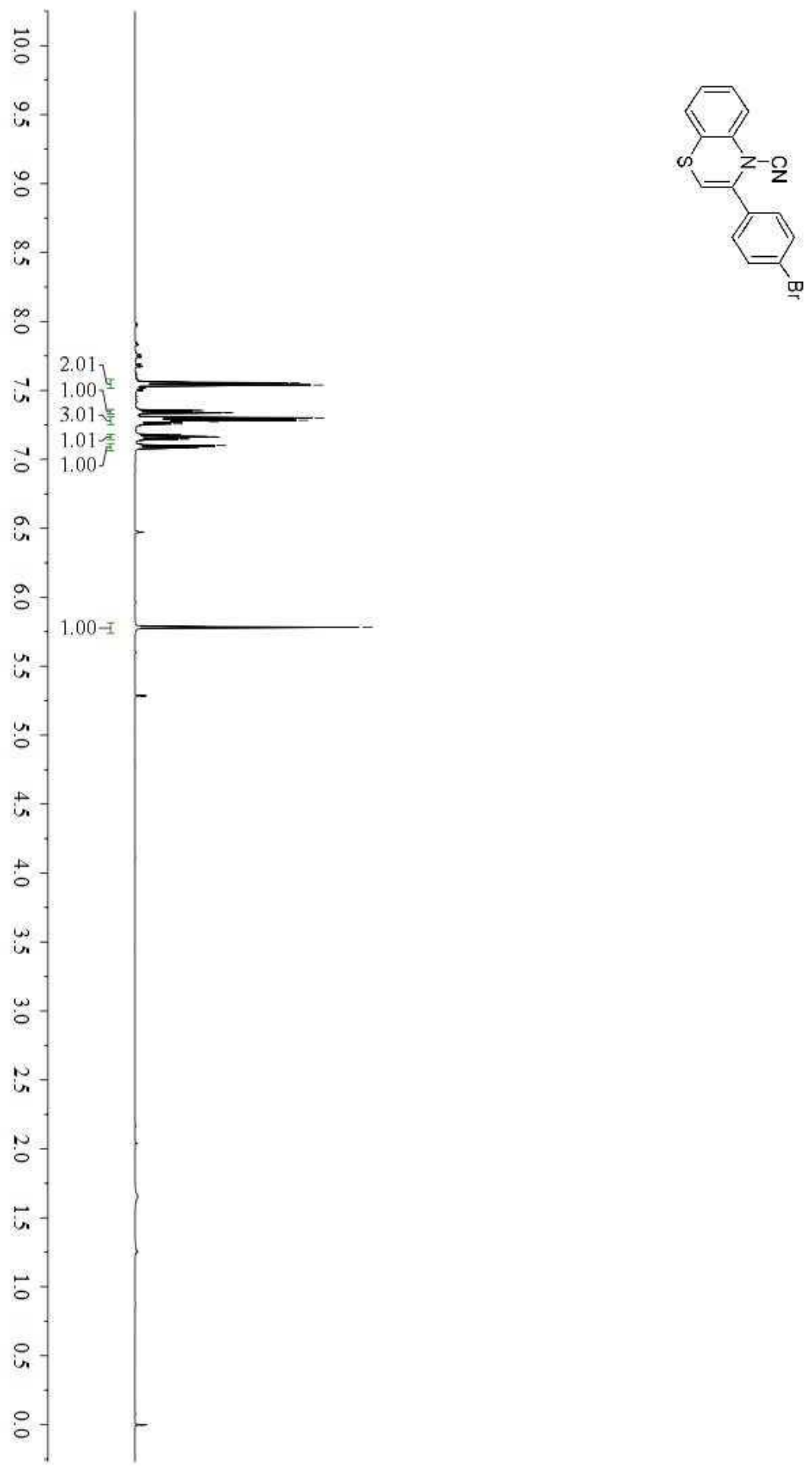

$-7.147$

$-7.100$

$-7.083$

$-5.783$ 
${ }^{13}$ C NMR of 3-(4-bromophenyl)-4H-benzo[b][1,4]thiazine-4-carbonitrile (3g)
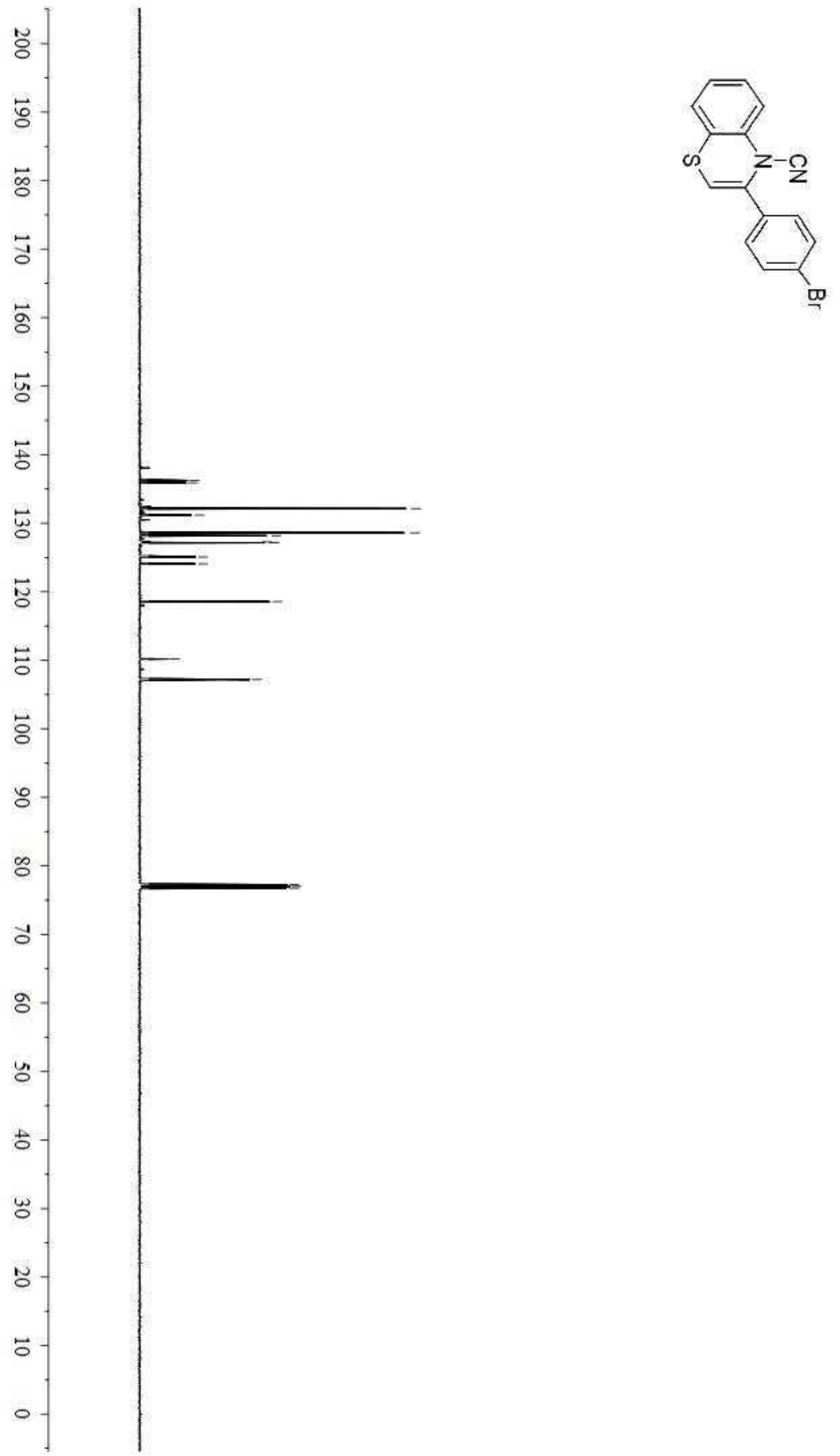

136.249

$-135.910$

$-132.139$

$-131.195$

- 128.604

- 128.184

1. 127.272

$-127.133$

125.074

$-124.085$

$-118.568$

$-110.176$

$-107.176$

77.254

$-77.000$

76.746 


\section{${ }^{1} \mathrm{H}$ NMR of}

3-(4-(trifluoromethyl)phenyl)-4H-benzo[b][1,4]thiazine-4-carbonitrile (3h)

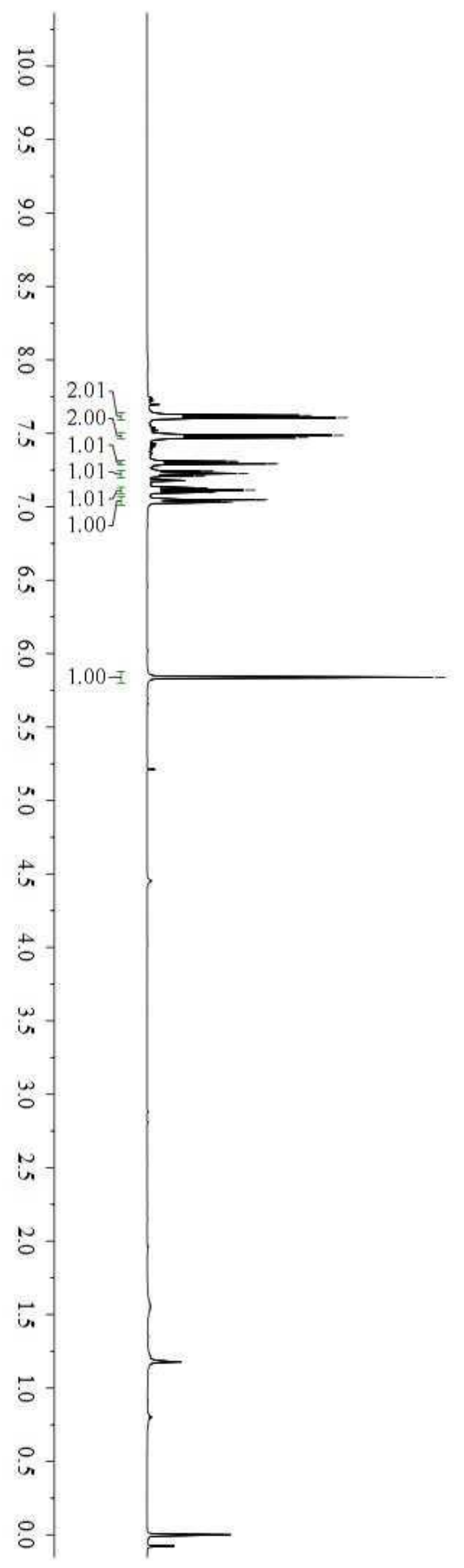

7.623

7.606

$-7.487$

$-7.470$

7.309
-7.308

$-7.293$

7.292

$-7.243$

$-7.241$

$-7.226$

$-7.213$

$-7.210$

7.129

7.127

$-7.114$

7.099

$-7.097$

$-7.048$

$-7.045$

$-7.032$

$-7.030$

$-5.839$ 


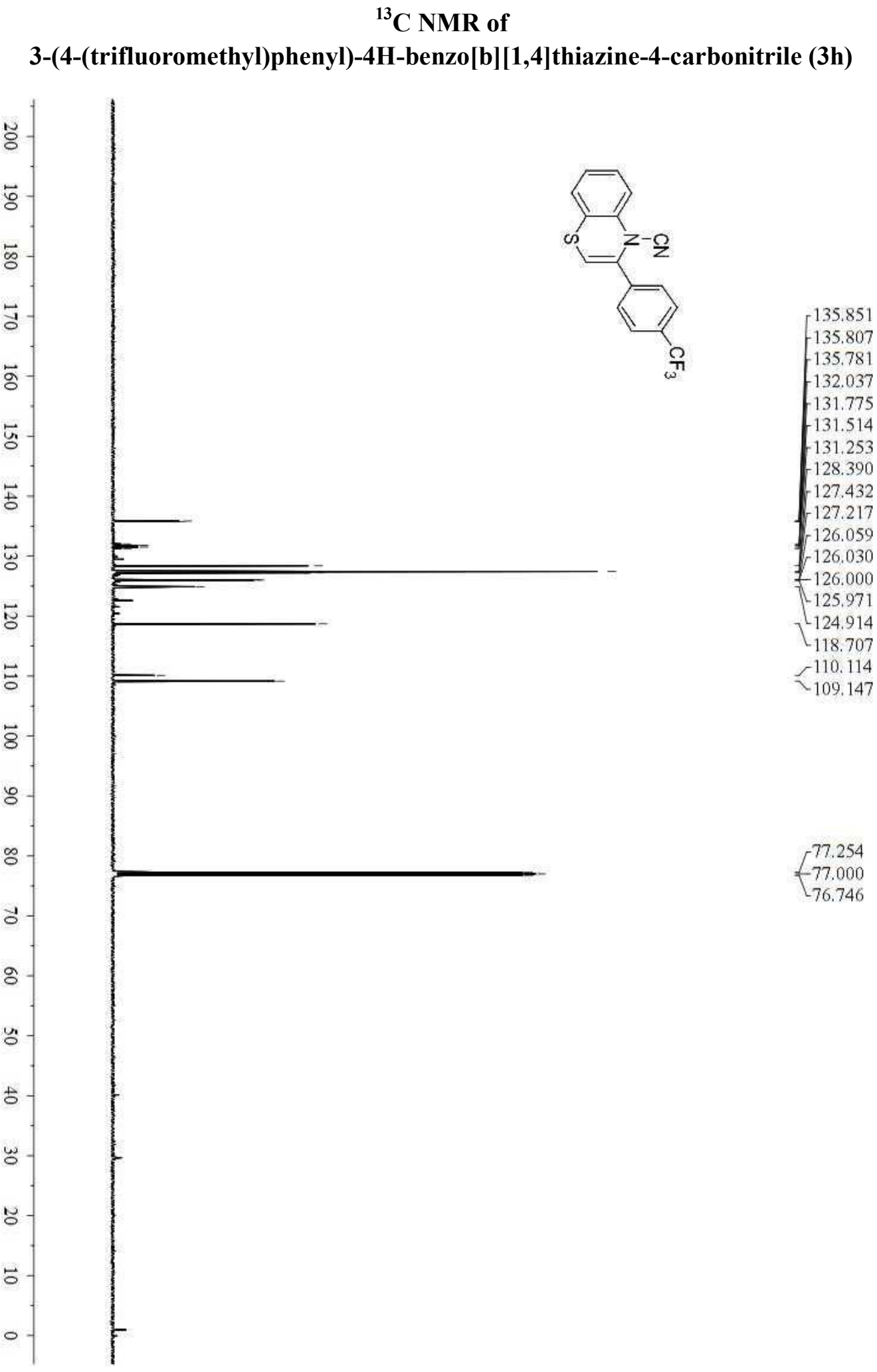


${ }^{1}$ H NMR of methyl 2-(4-cyano-4H-benzo[b] [1,4]thiazin-3-yl)benzoate (3i)
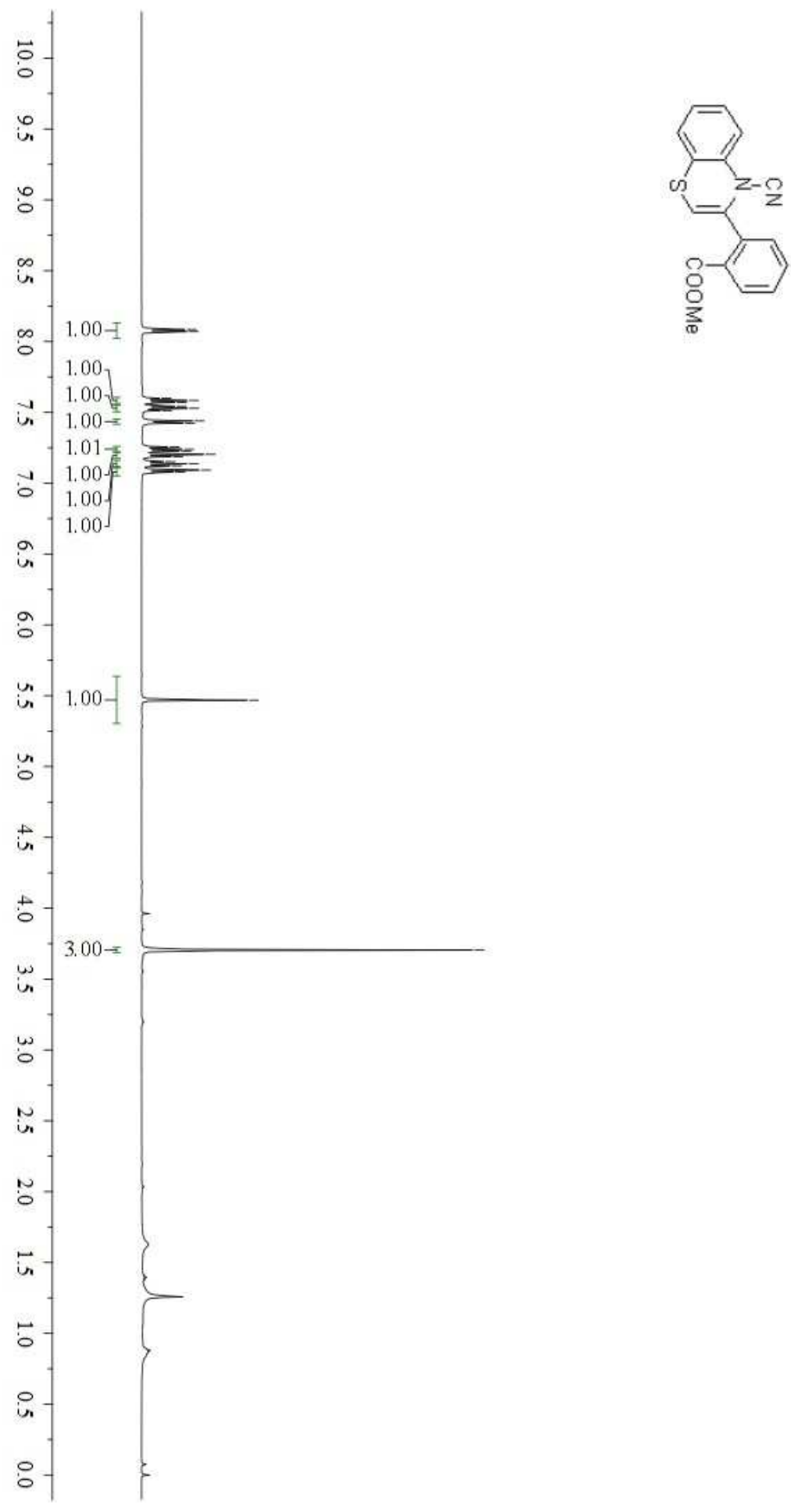

$<.085$

7.584

$-7.543$

$-7.513$

$-7.424$

7.239

7.203

$-7.152$

$-7.122$

7.078

$-5.469$

$-3.705$ 
${ }^{13}$ C NMR of methyl 2-(4-cyano-4H-benzo[b] [1,4]thiazin-3-yl)benzoate (3i)

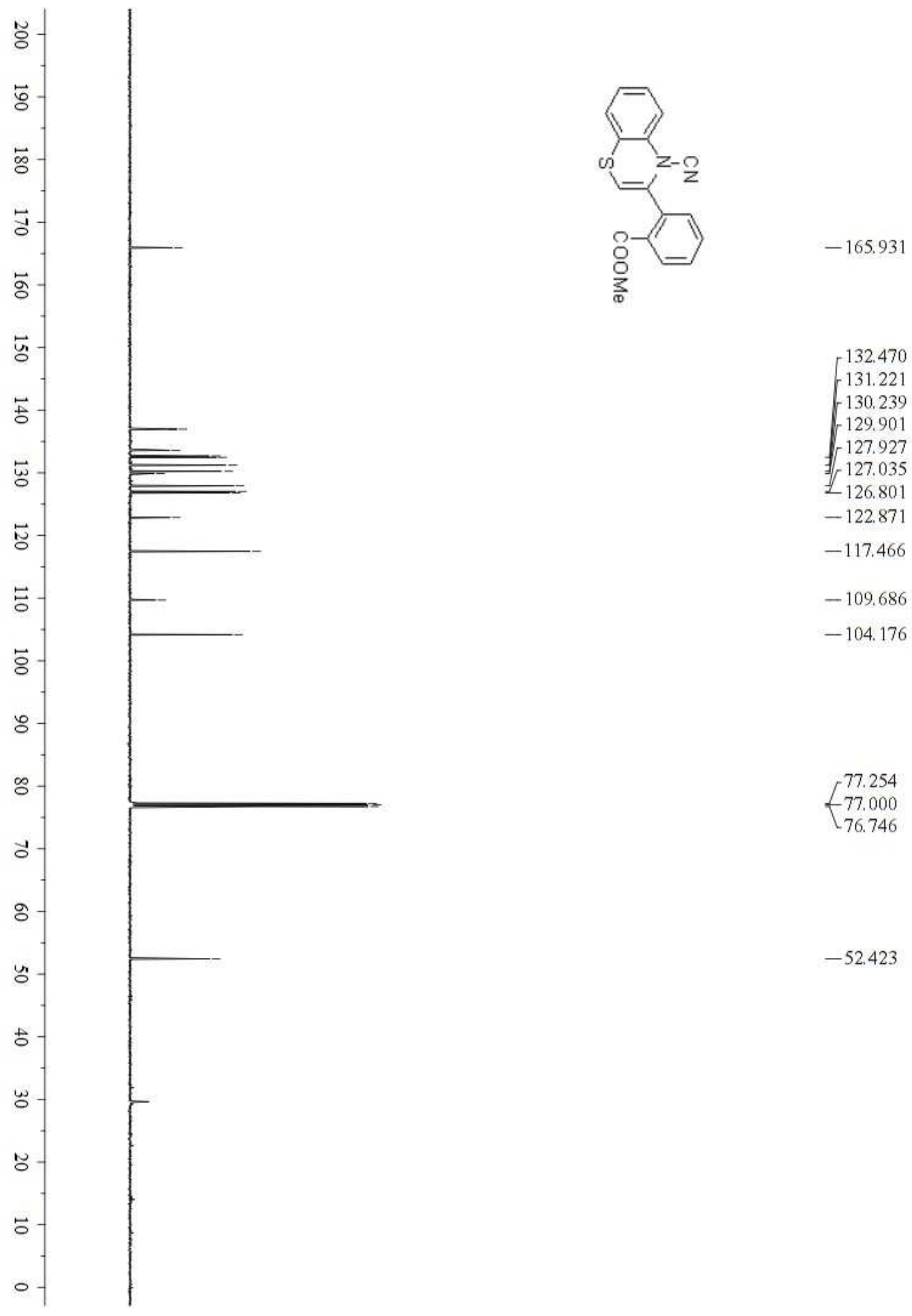


${ }^{1}$ H NMR of 3-(p-tolyl)-4H-benzo[b][1,4]thiazine-4-carbonitrile (3j)
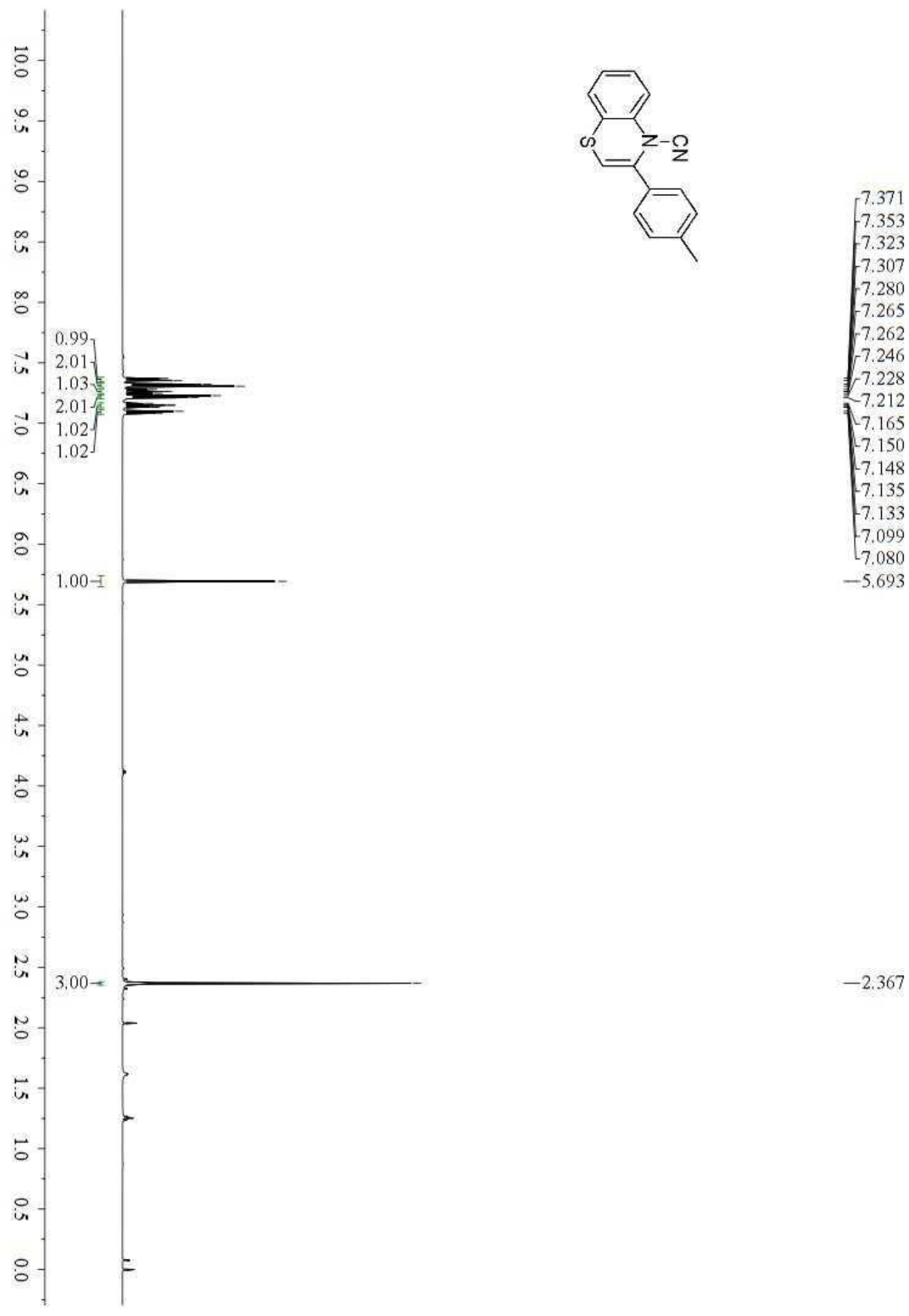


\section{${ }^{13}$ C NMR of 3-(p-tolyl)-4H-benzo[b] [1,4]thiazine-4-carbonitrile (3j)}

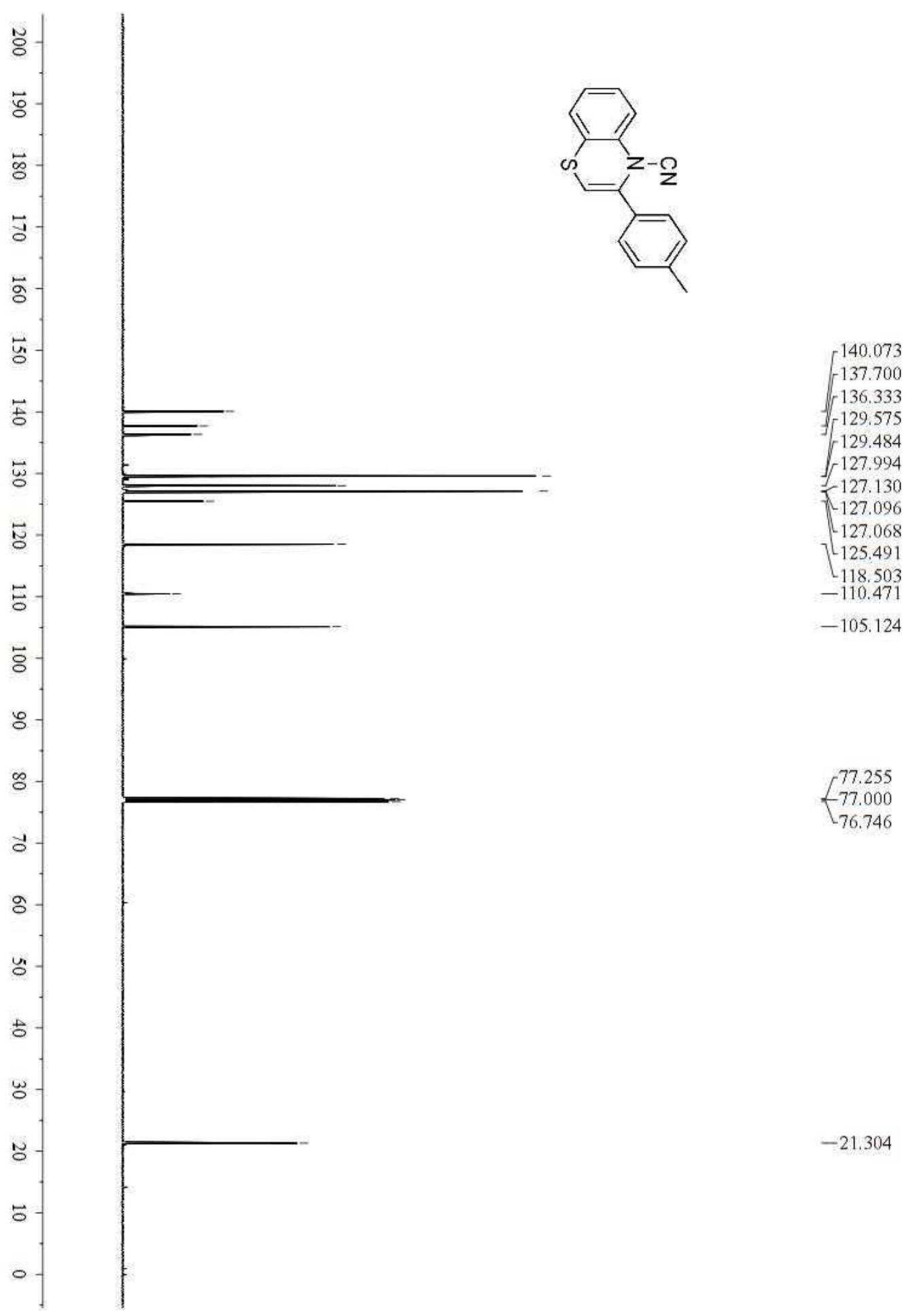


${ }^{1}$ H NMR of 3-(m-tolyl)-4H-benzo[b][1,4]thiazine-4-carbonitrile (3k)
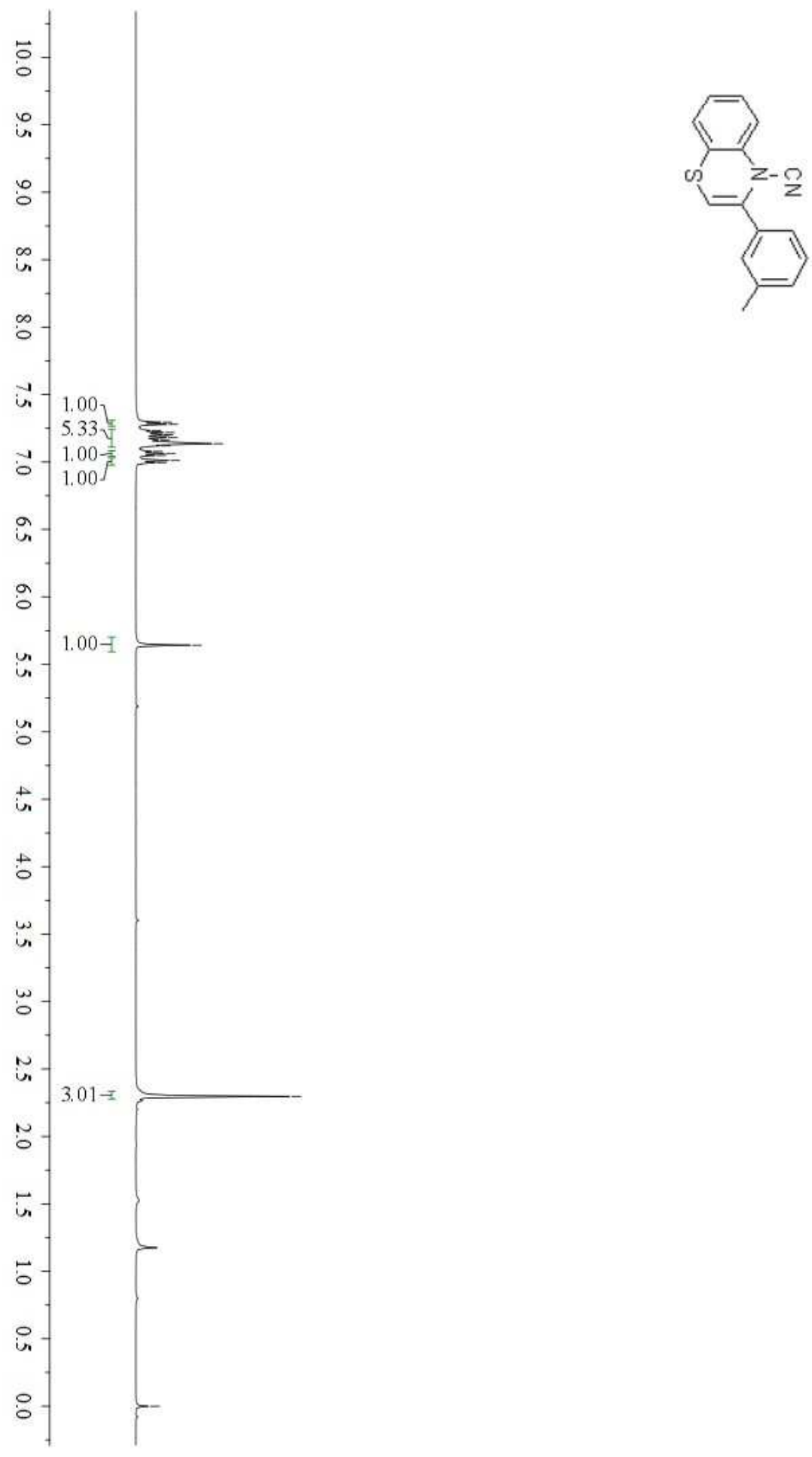

7.295
-7.279
-7.233

$-7.218$

$-7.202$

$-7.196$

$-7.180$

$-7.164$

7.136

$-7.120$

$-7.078$

$-7.063$

$-7.048$

$-7.011$

6.996

$-5.642$

$-2.297$

$-0.000$ 


\section{${ }^{13} \mathrm{C}$ NMR of 3-(m-tolyl)-4H-benzo[b][1,4]thiazine-4-carbonitrile (3k)}

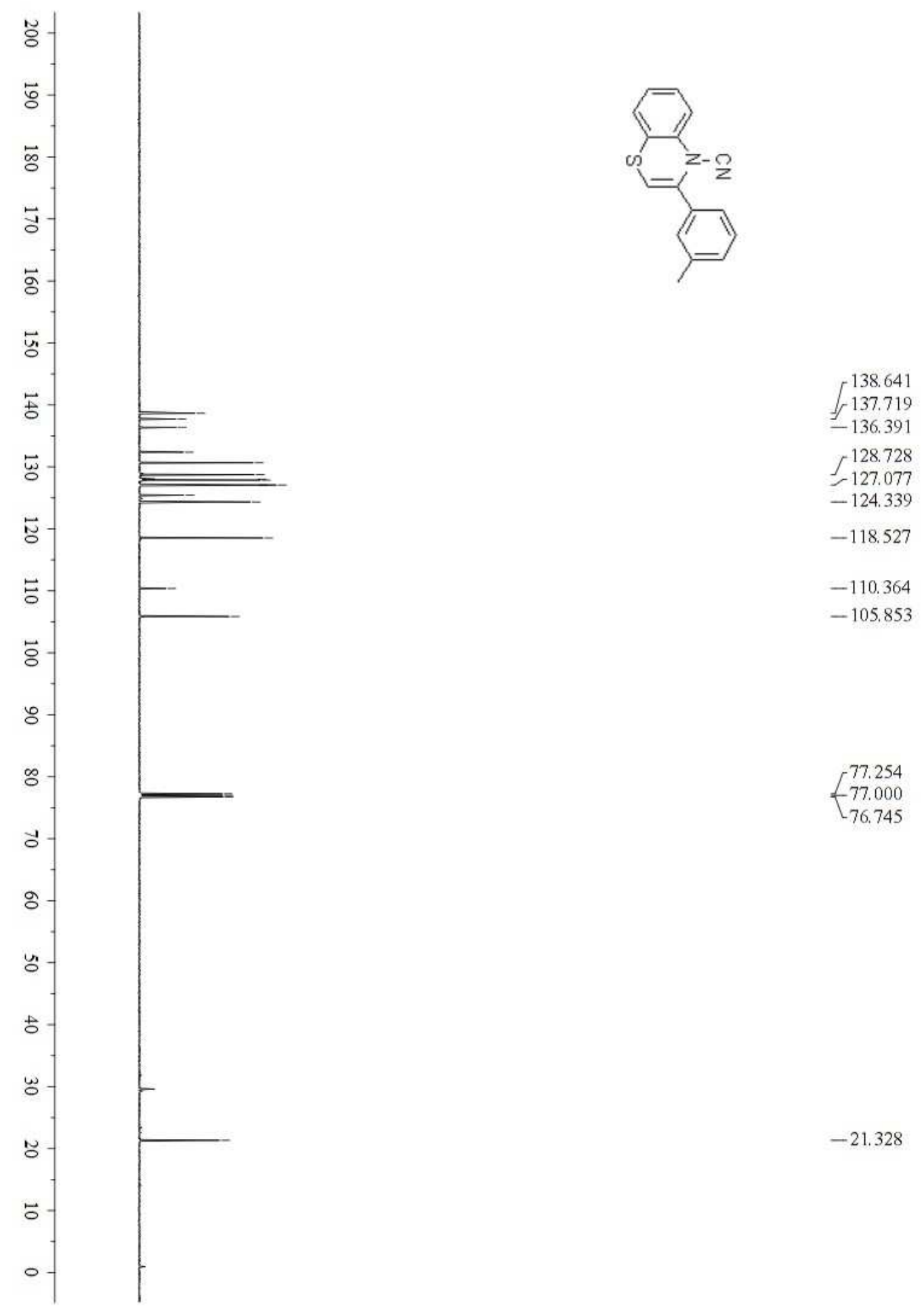


${ }^{1}$ H NMR of 3-(4-ethylphenyl)-4H-benzo[b][1,4]thiazine-4-carbonitrile (3I)

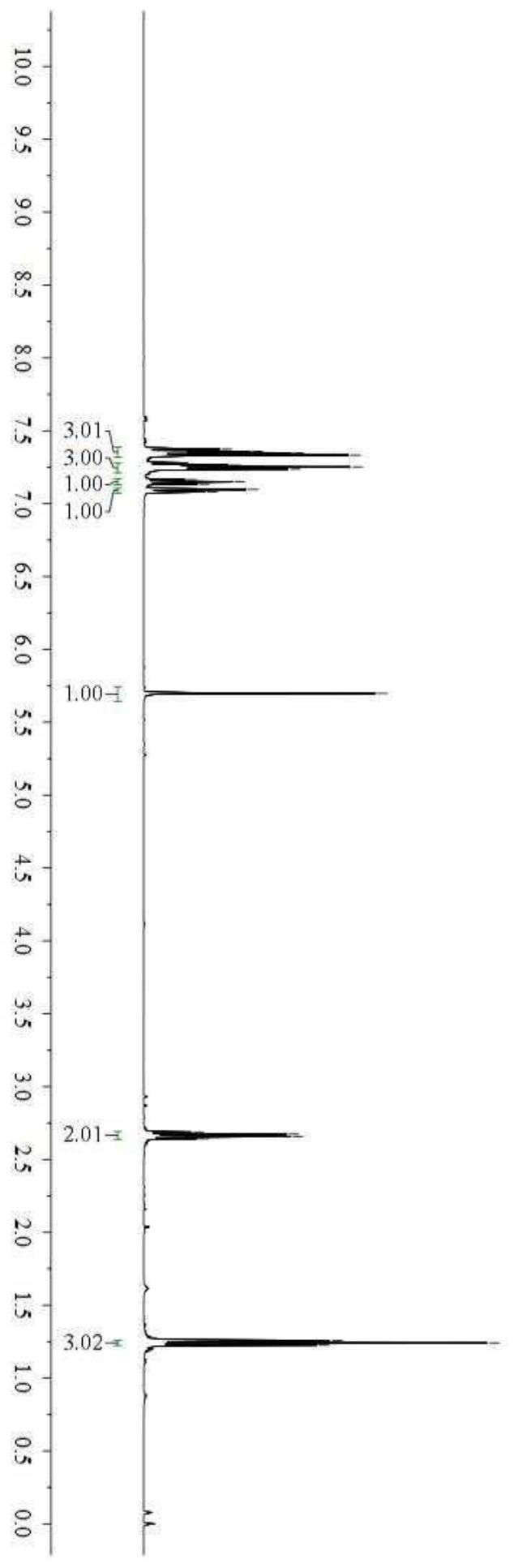

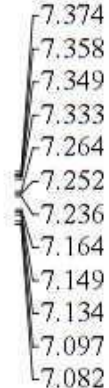

$-5.698$

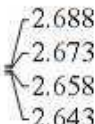

$-1.242$

$-1.242$ 
${ }^{13}$ C NMR of 3-(4-ethylphenyl)-4H-benzo[b] [1,4]thiazine-4-carbonitrile (3l)

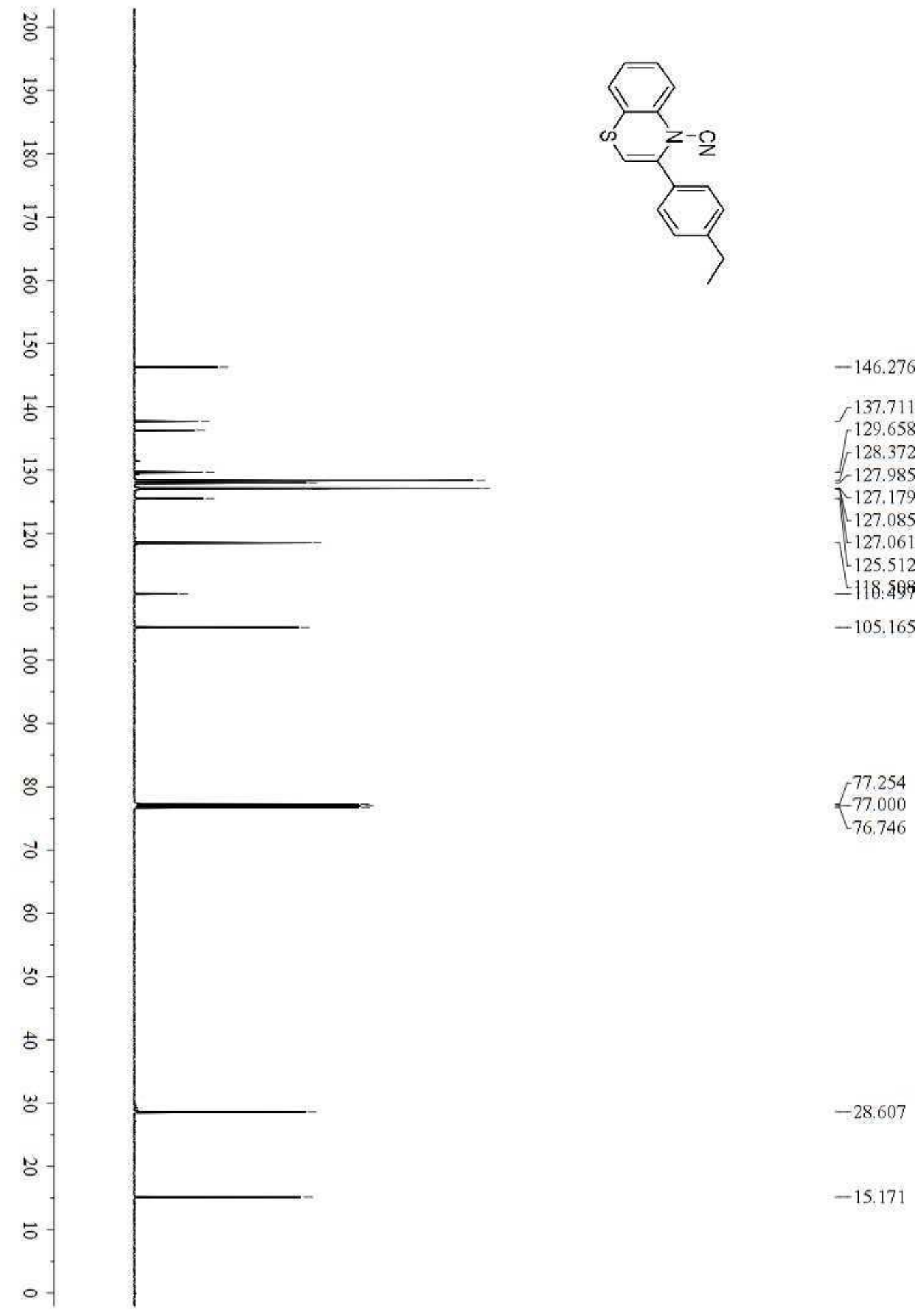


${ }^{1}$ H NMR of 3-(4-propylphenyl)-4H-benzo[b][1,4]thiazine-4-carbonitrile (3m)
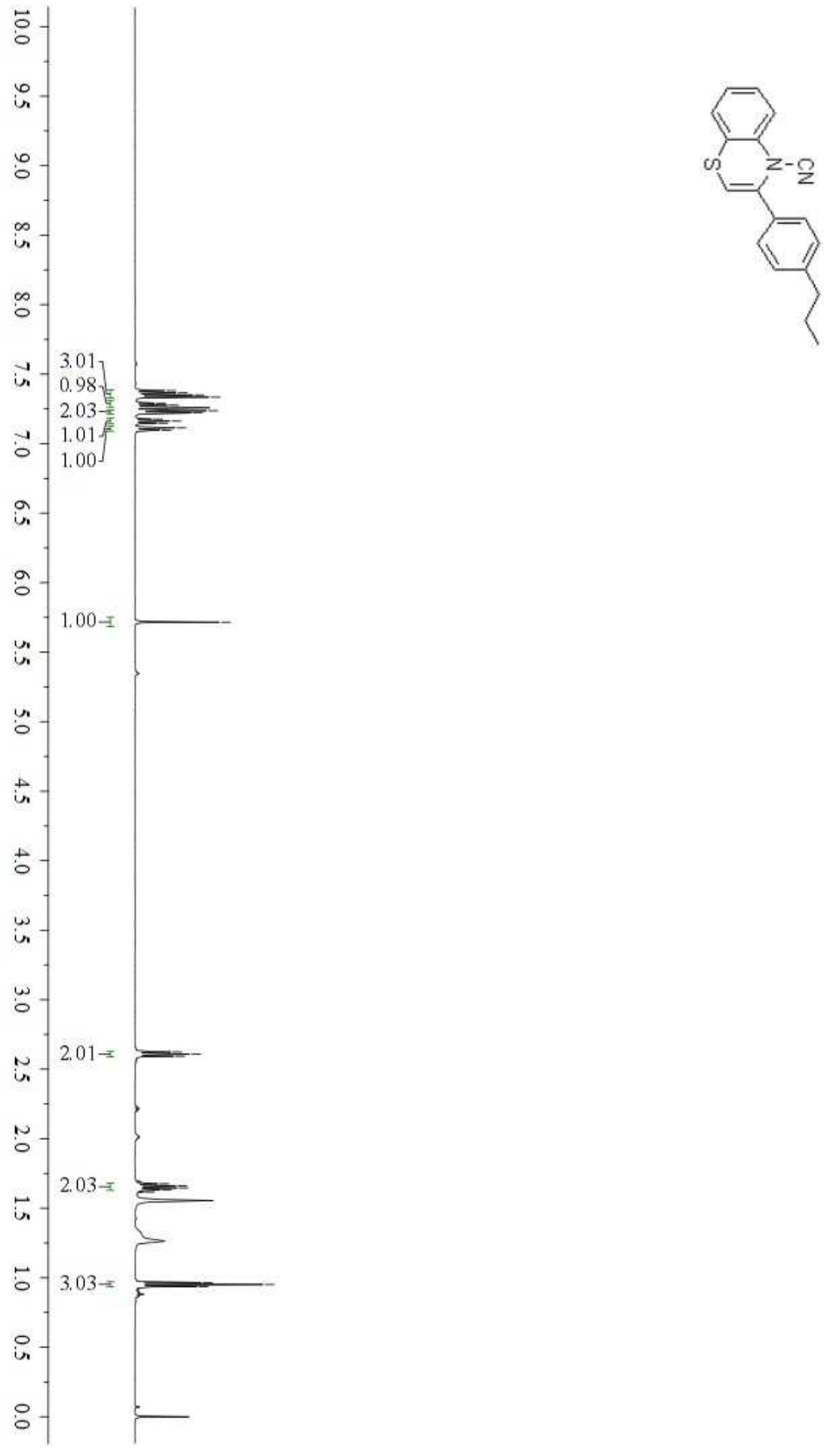

$\left[\begin{array}{r}7.382 \\ 7.366 \\ -7.351 \\ 7.334 \\ 7.292 \\ 7.277 \\ 7.237 \\ 7.221 \\ -7.177 \\ 7.162 \\ 7.147 \\ 7.113 \\ 7.098\end{array}\right.$

$-5.716$

2.624

$-2.609$

-2.593

1.676

1.661

$-1.646$

$-1.631$

$-1.617$

$\left\{\begin{array}{r}0.966 \\ -0.951\end{array}\right.$

$\iota_{0.936}$ 
${ }^{13}$ C NMR of 3-(4-propylphenyl)-4H-benzo[b] [1,4]thiazine-4-carbonitrile (3m)
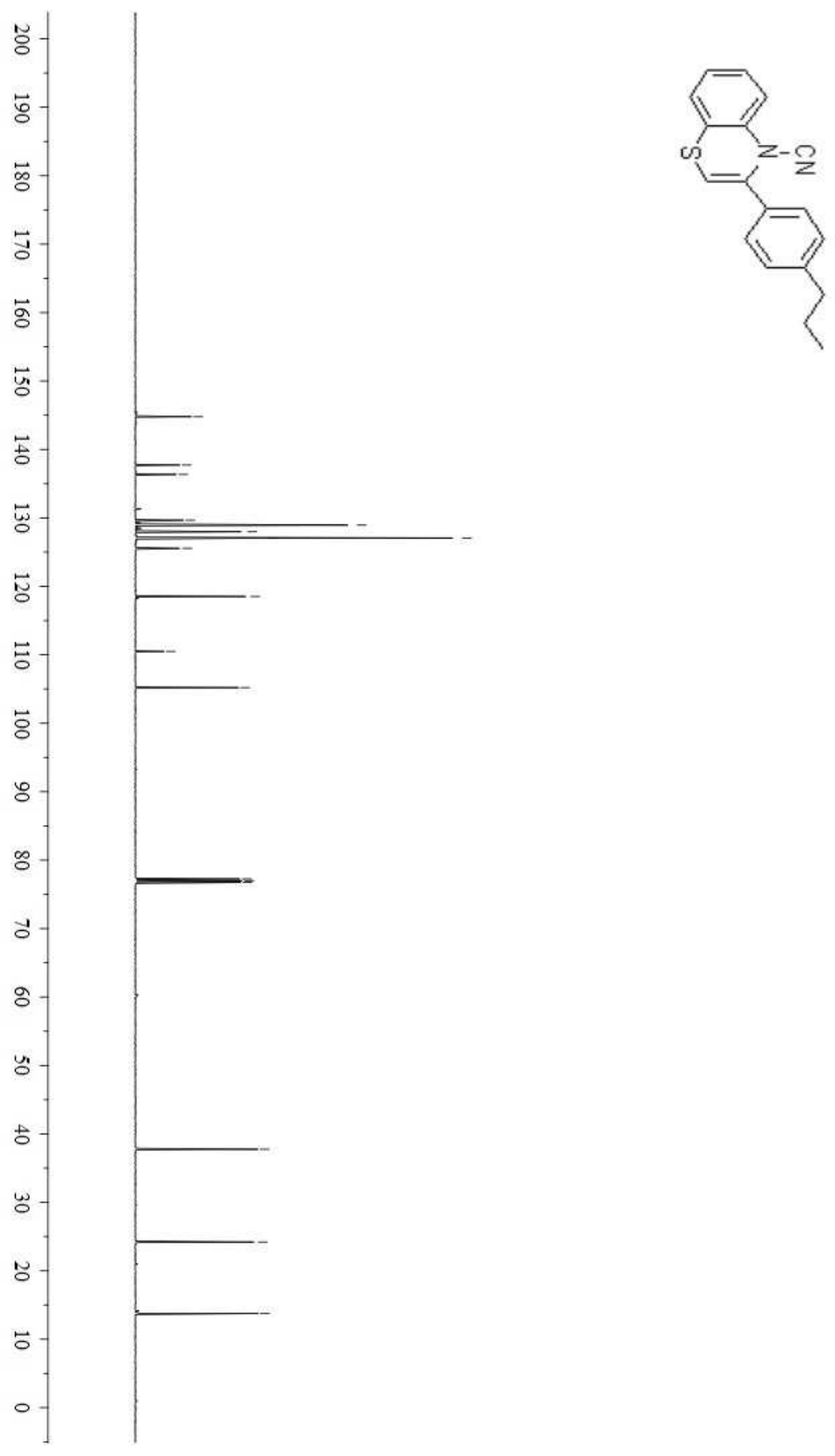

$-144.785$

$-136.352$

$-127.977$

$-125.553$

$-118.519$

$-110.490$

$-105.202$

77.254

$-77.000$

$-76.746$

$-37.752$

$-24.195$

$-13.763$ 
${ }^{1}$ H NMR of 3-(4-butylphenyl)-4H-benzo[b] [1,4]thiazine-4-carbonitrile (3n)

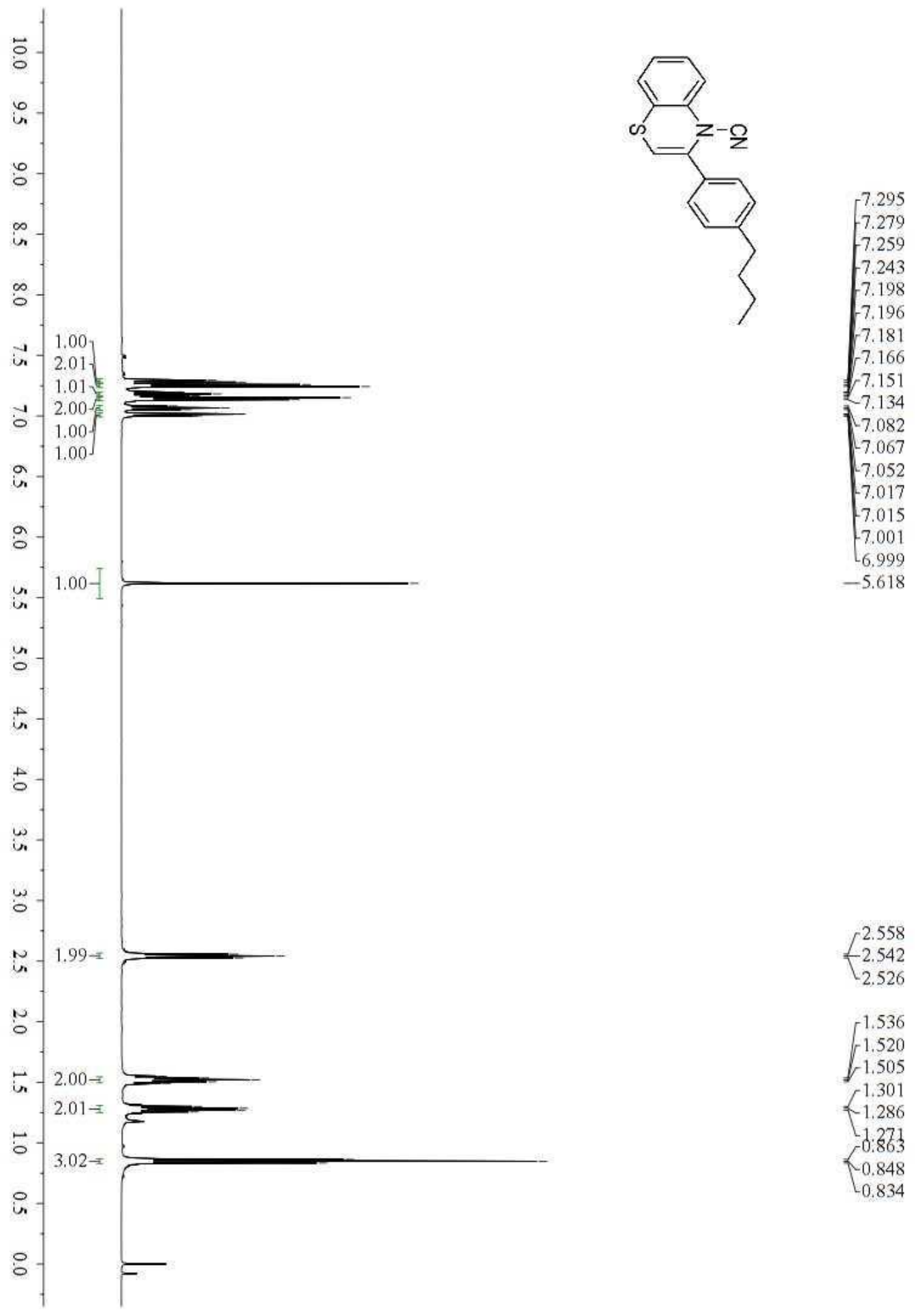


${ }^{13}$ C NMR of 3-(4-butylphenyl)-4H-benzo[b] [1,4]thiazine-4-carbonitrile (3n)

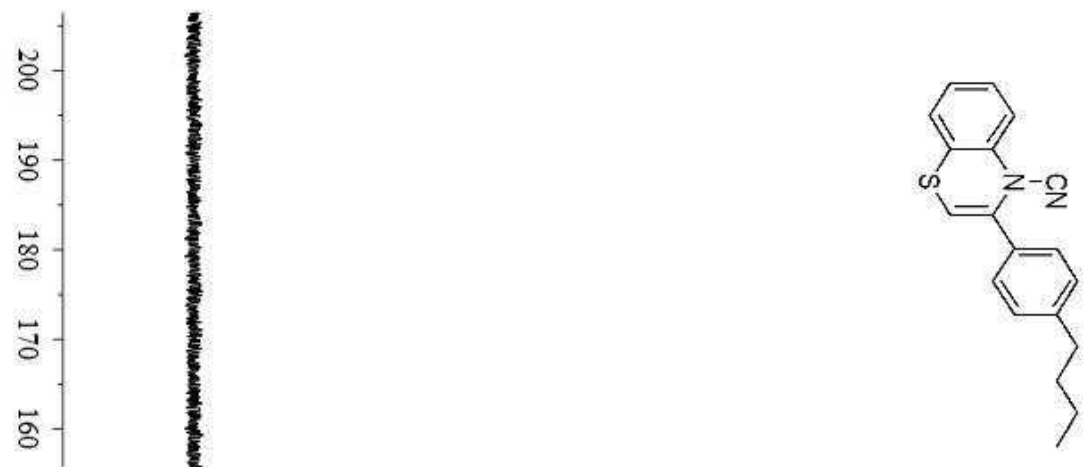

145.024

137.743

136.364

] 129.631

128.895

- 127.979

127.090

127.053

125.560

118.525

$-110.491$

$-105.182$

후

8

\&

o

8

प̆

b

w

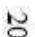

ㅎ

$\circ$

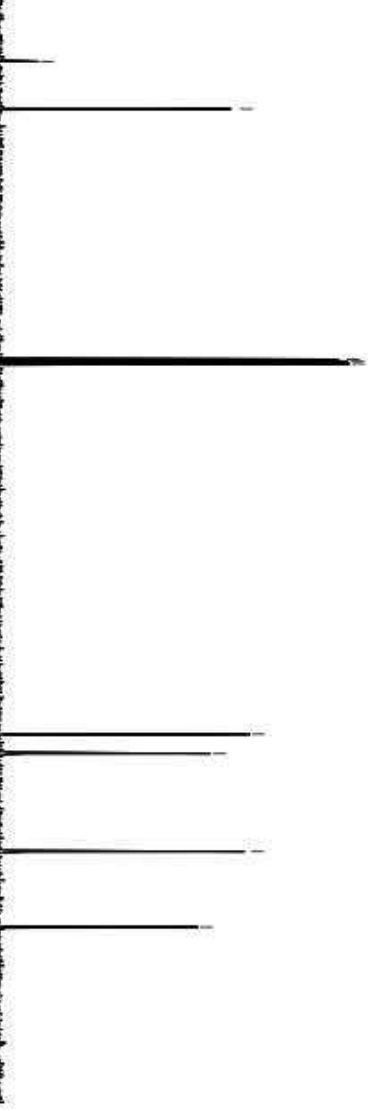

77.253

$-77.000$

76.745

$-35.387$

$-33.230$

$-22.288$

$-13.857$ 


\section{${ }^{1}$ H NMR of 3-(4-(tert-butyl)phenyl)-4H-benzo[b][1,4]thiazine-4-carbonitrile (3o)}

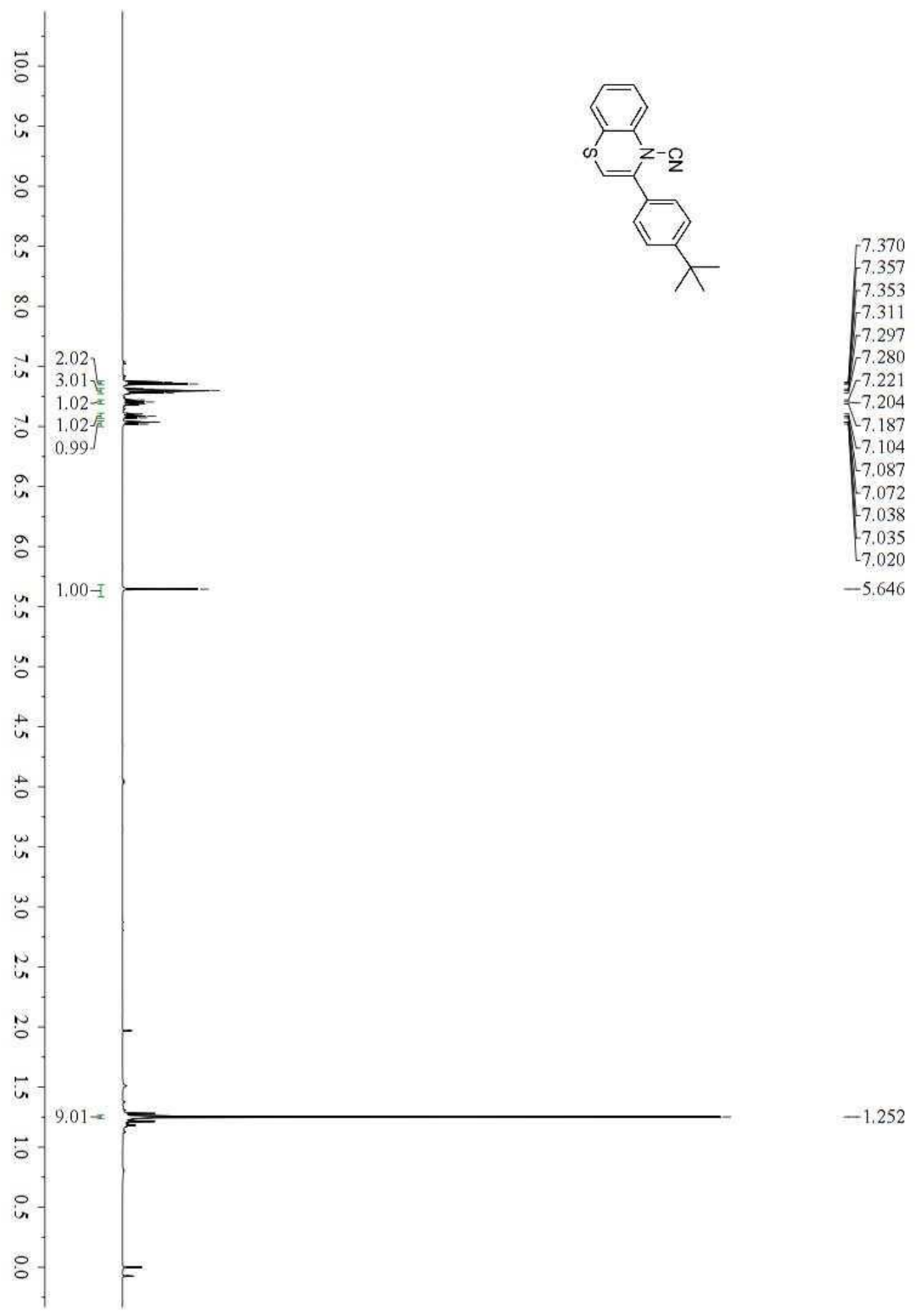


${ }^{13}$ C NMR of 3-(4-(tert-butyl)phenyl)-4H-benzo[b][1,4]thiazine-4-carbonitrile (3o)
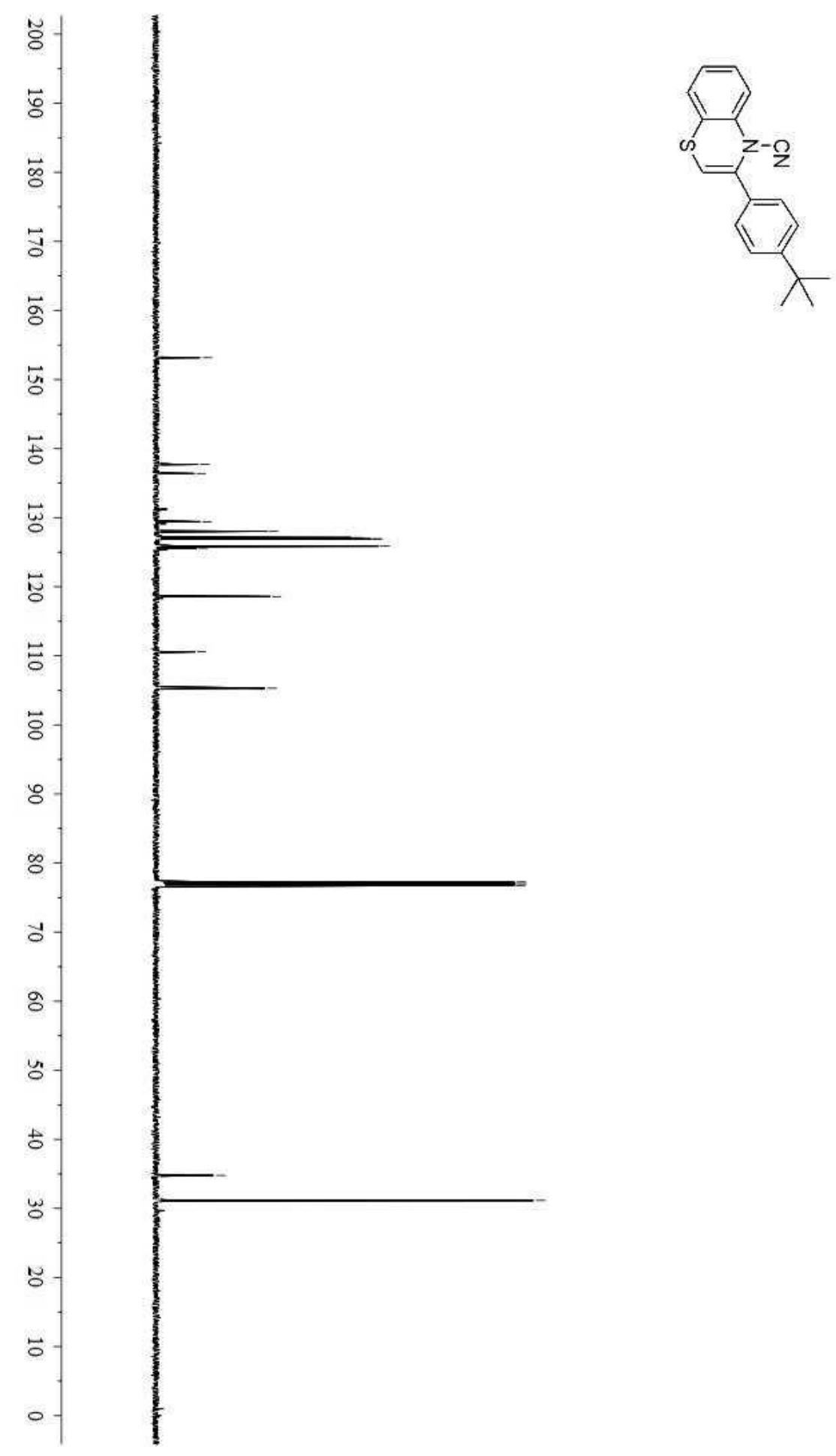

$-153.165$

137.699

136.394

129.418

128.036

127.113

$-126.930$

-126.921

$-125.873$

$-125.602$

118.586
-110.577

$-105.310$

77.254

$=77.000$

$-76.747$

$-34.779$

$-31.157$ 
${ }^{1}$ H NMR of 3-(4-methoxyphenyl)-4H-benzo[b] [1,4] thiazine-4-carbonitrile (3p)

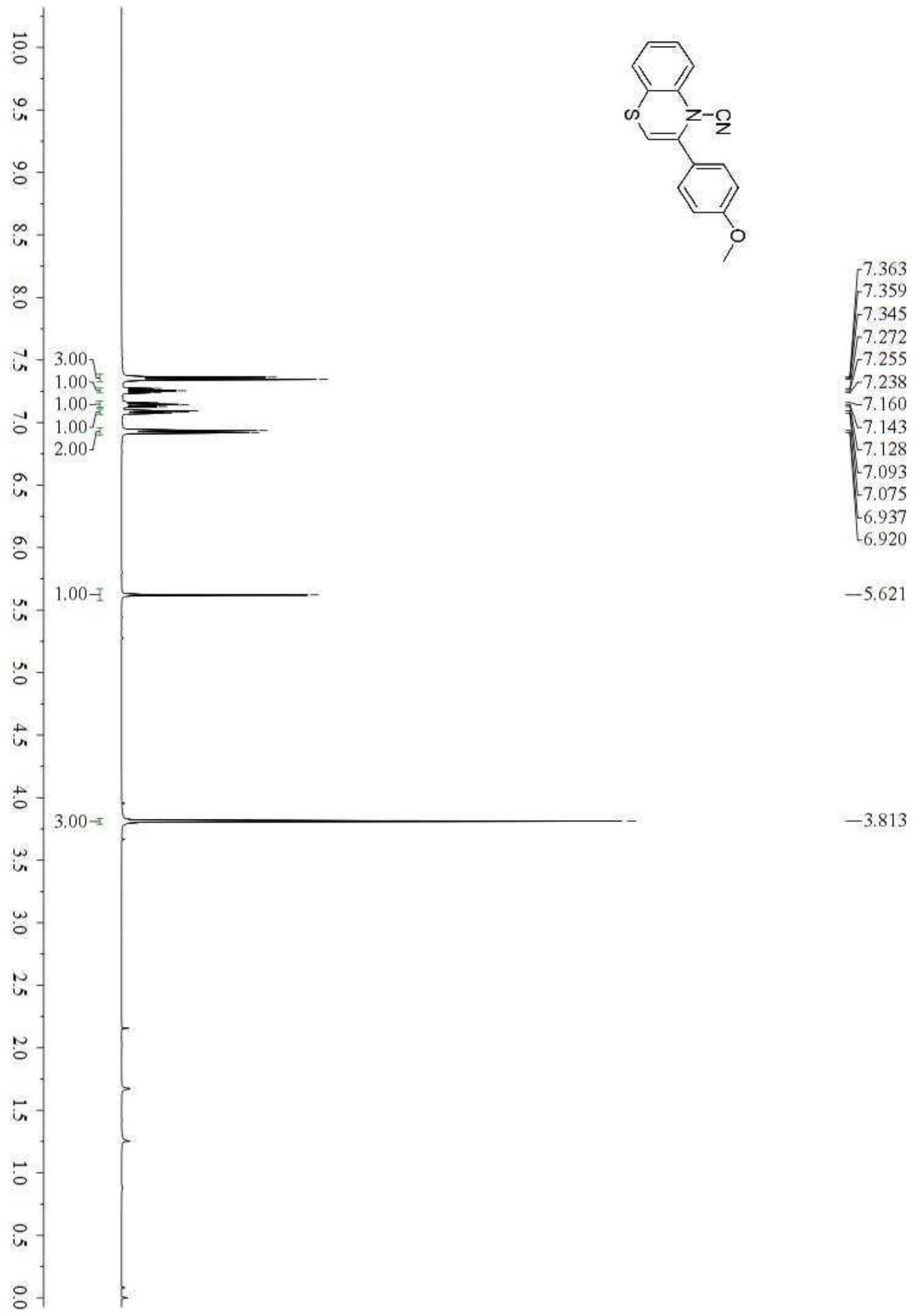


${ }^{13}$ C NMR of 3-(4-methoxyphenyl)-4H-benzo[b][1,4]thiazine-4-carbonitrile (3p)

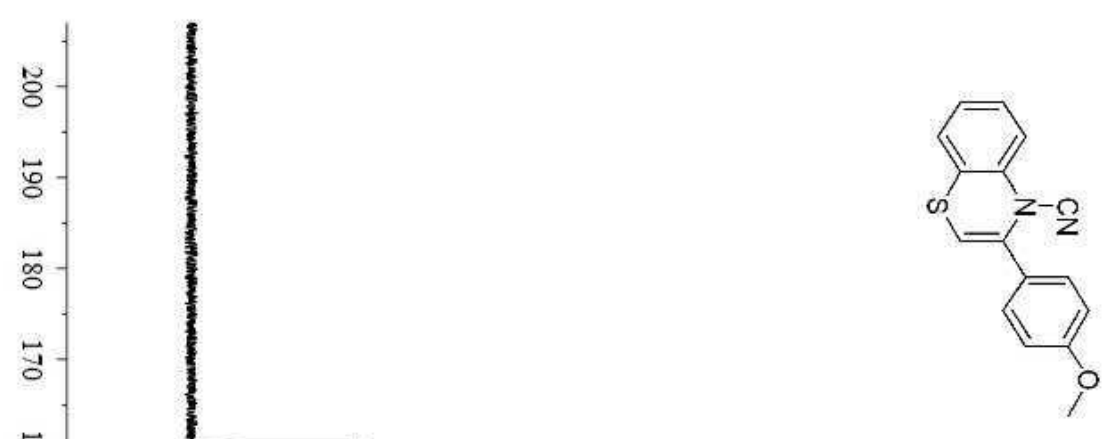

$-160.767$

जั

点

항

뭉

节

횽

\&

$\infty$

ชี

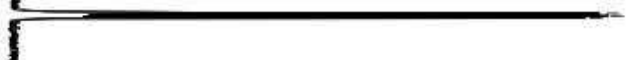

8

光

b

$\ddot{\circ}$

ก

5

$\circ-$

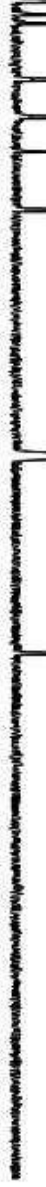

77.255

$-77.000$

$-76.746$

$-137.553$

136,300

128.693

127.914

$-127.072$

125.614

124.593

118.454

$-114.257$

$-110.503$

$-104.093$

$-55.285$ 
${ }^{1}$ H NMR of 3-(thiophen-3-yl)-4H-benzo[b] [1,4]thiazine-4-carbonitrile (3q)

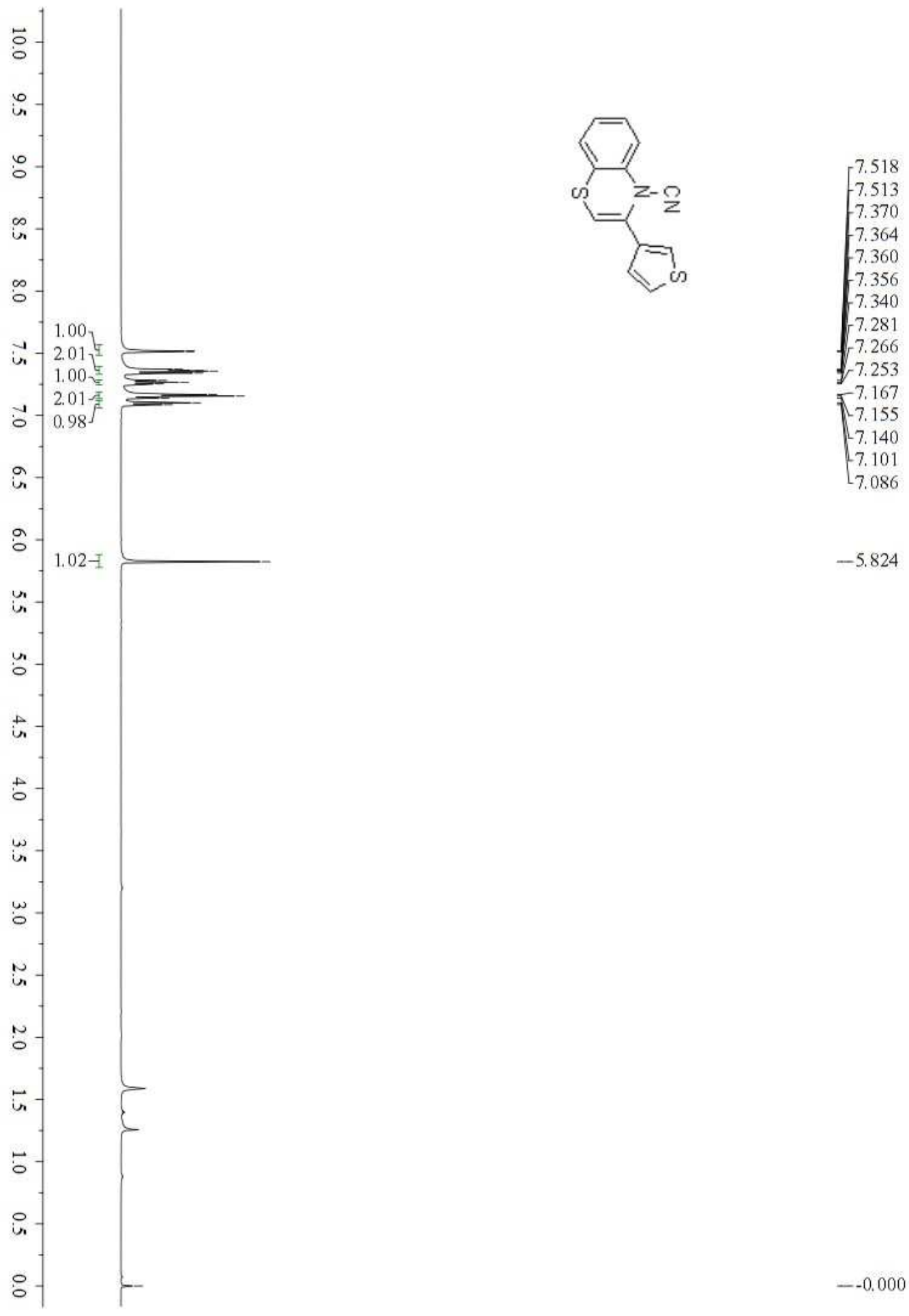




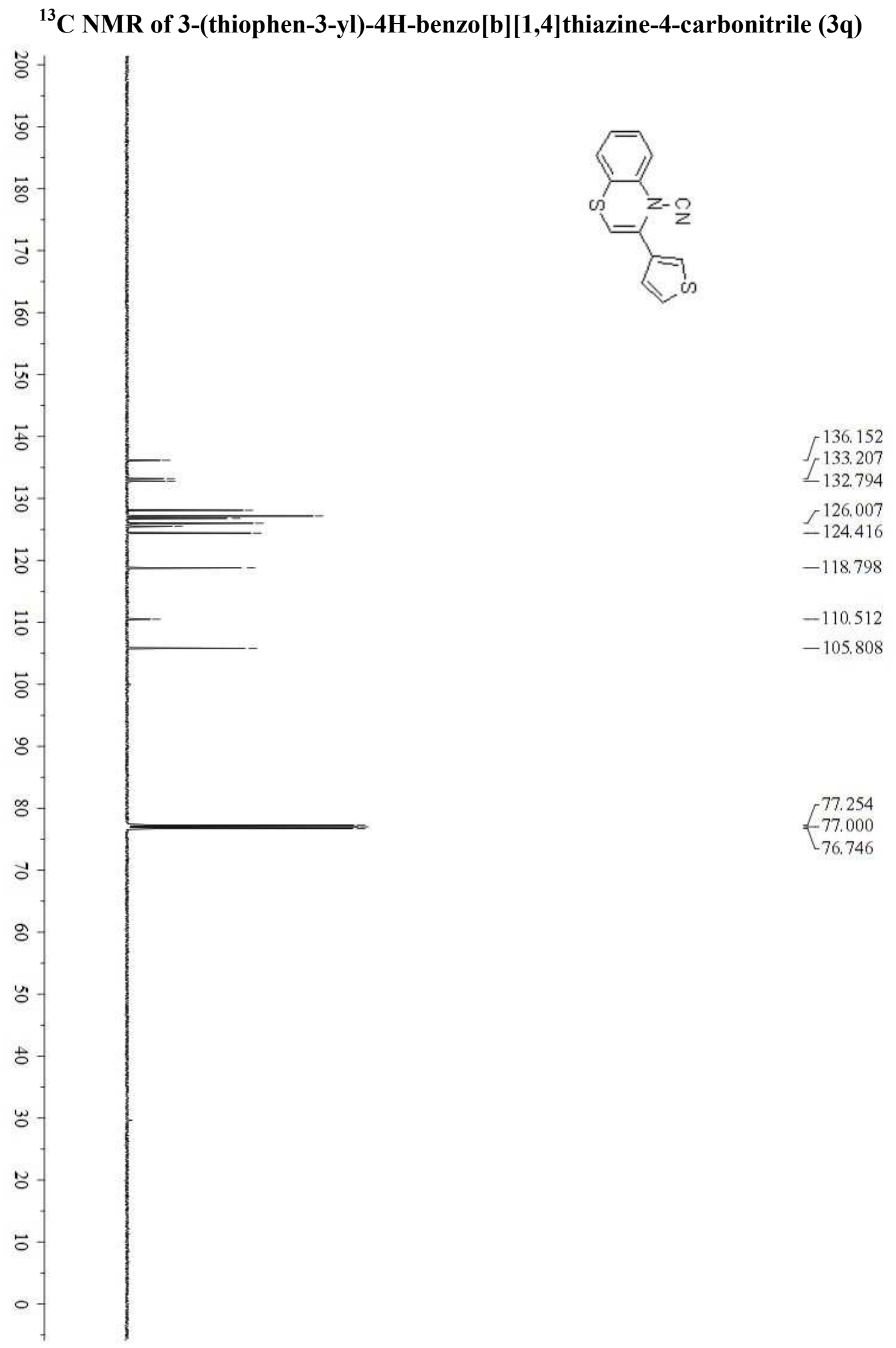


${ }^{1}$ H NMR of 3-(cyclohex-1-en-1-yl)-4H-benzo[b][1,4]thiazine-4-carbonitrile (3r)
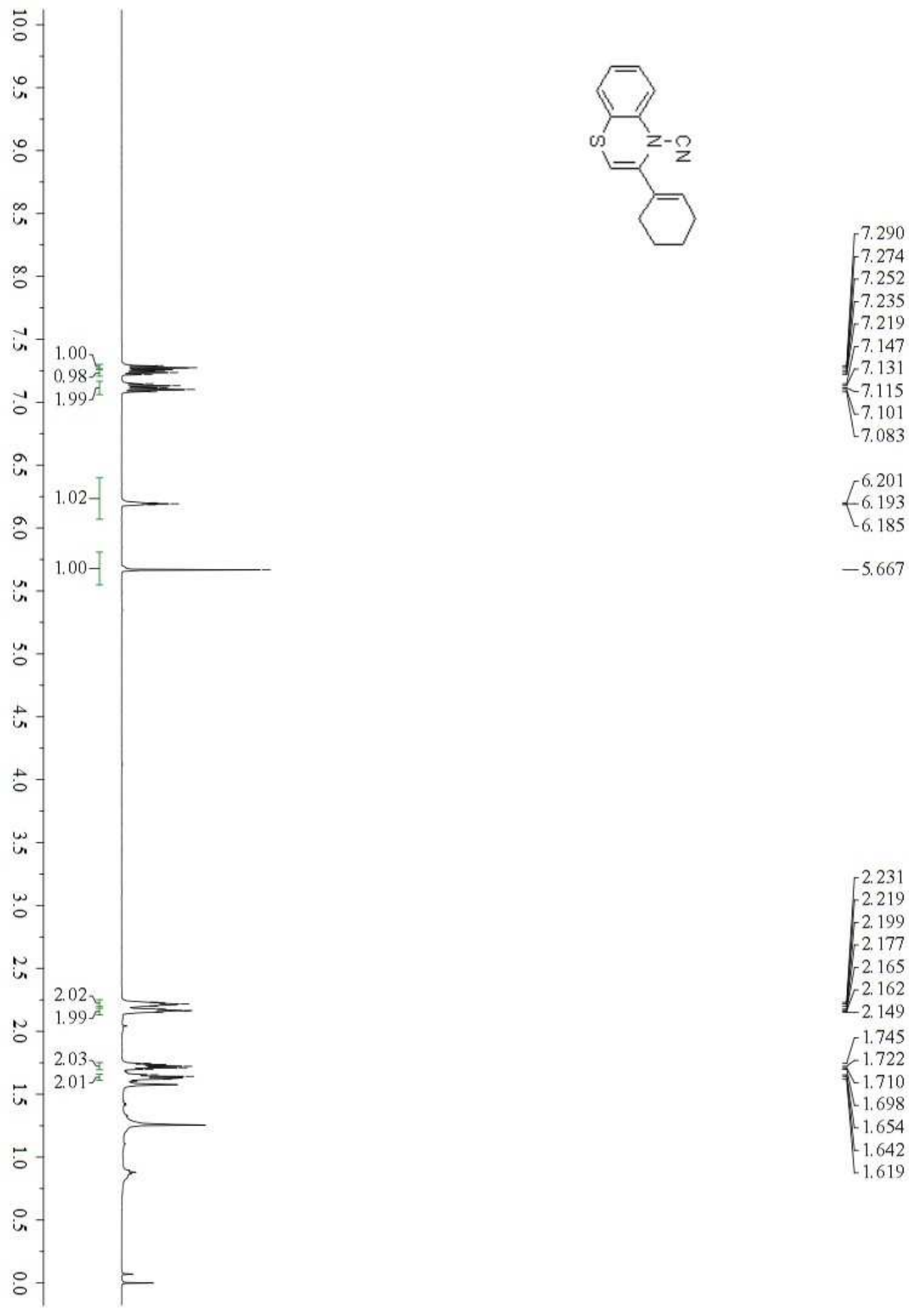

2.231

$-2.219$

$-2.199$

$-2.177$

- 2.165

$-2.162$

$-2.149$

$-1.745$

- 1.722

$-1.710$

1.698

$-1.654$

$-1.642$

[1.619 


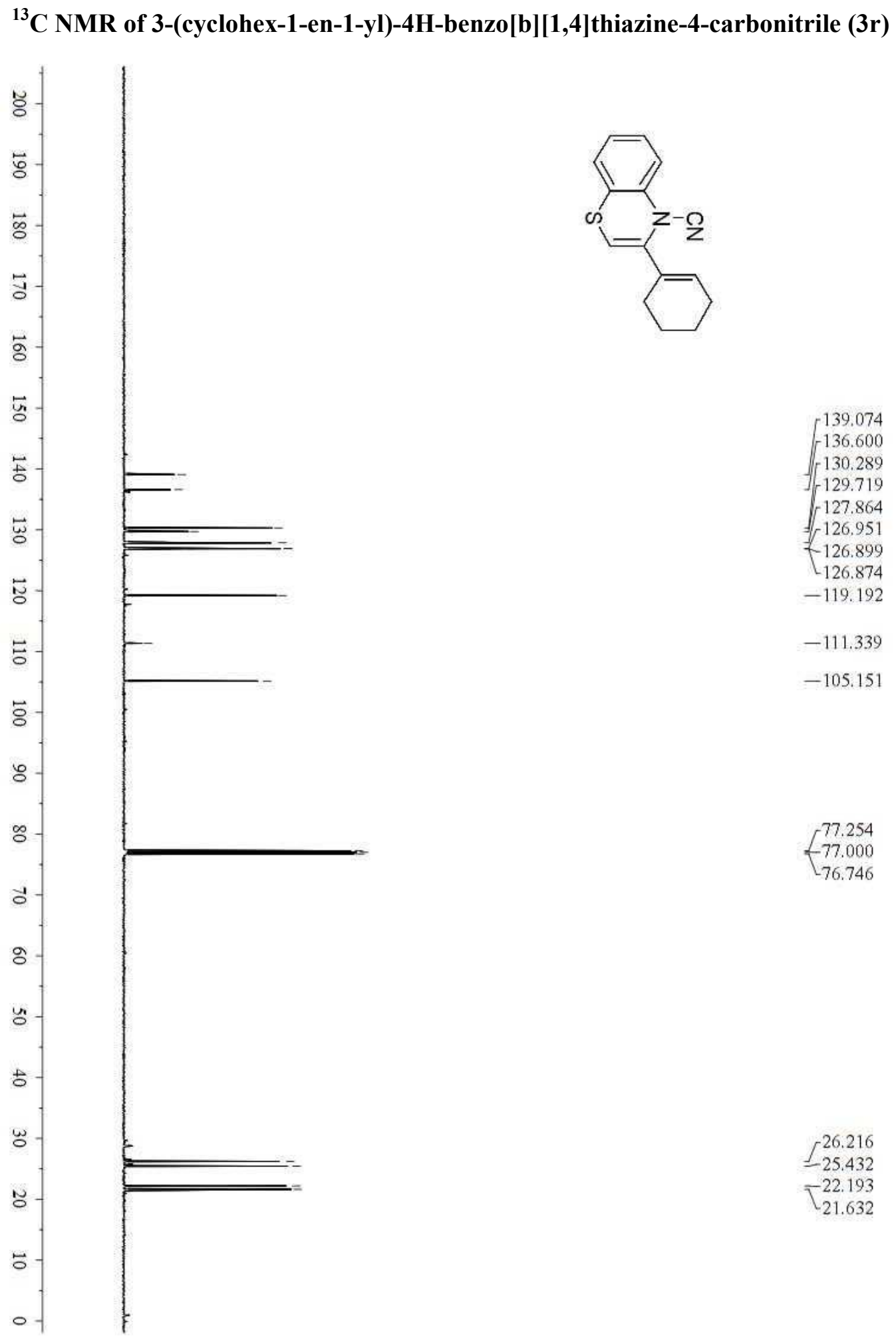


${ }^{1} \mathrm{H}$ NMR of 3-octyl-4H-benzo[b][1,4]thiazine-4-carbonitrile (3s)
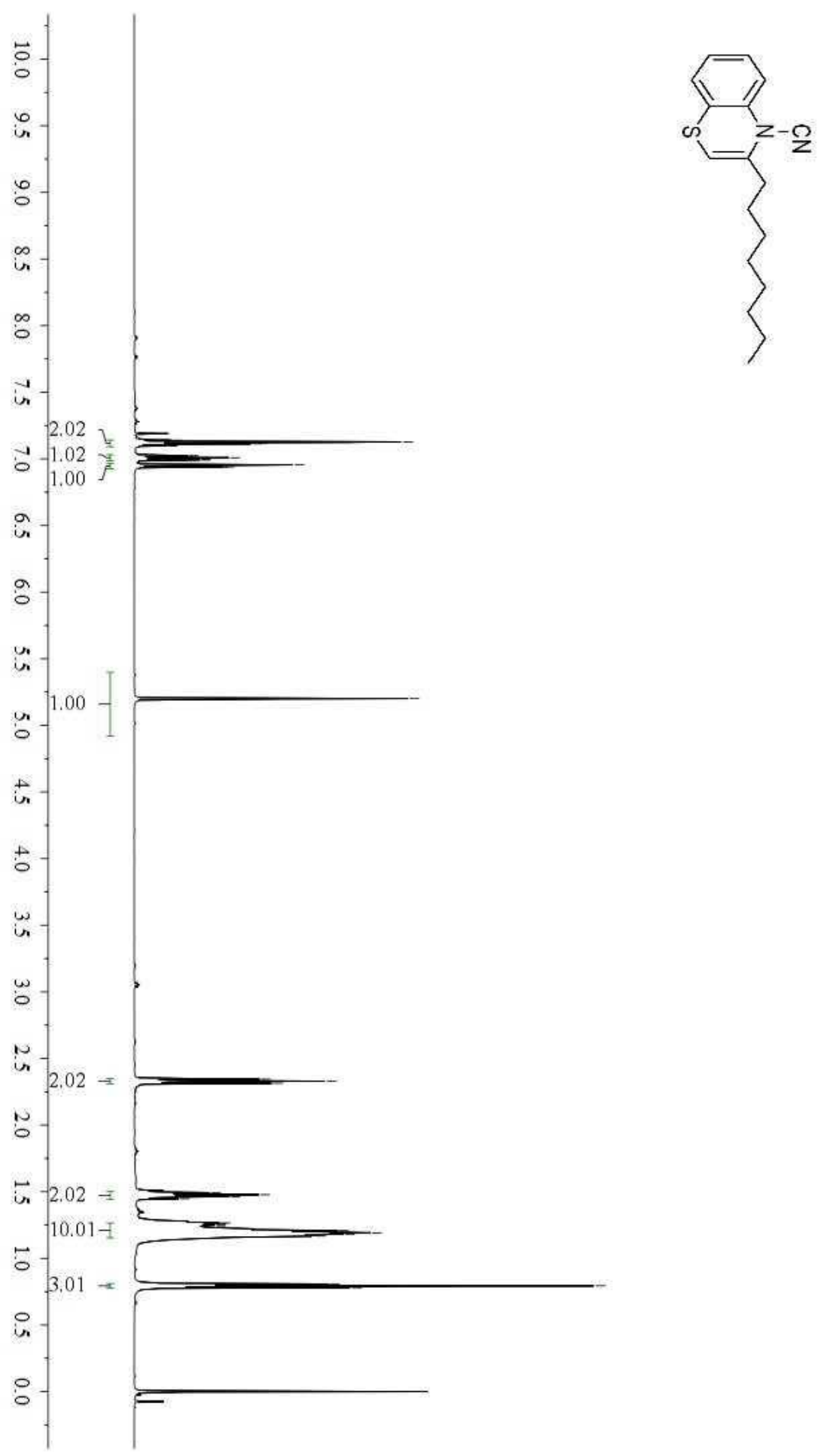

7.140
-7.128
-7.126
-7.124
7.116
7.113
7.100
-7.097
7.024
7.019
7.011
7.008
-7.004
-6.996
-6.991
-6.954
6.940
6.938
-5.201

2.344
-2.329

- 2.314

$\int_{-1.478}^{1.463}$

1.265

1.261

1.236

$-1.208$

$-1.192$

$-1.182$

$-1.172$

$-1.167$

$-0.807$

$-0.794$

$-0.780$ 
${ }^{13} \mathrm{C}$ NMR of 3-octyl-4H-benzo[b][1,4]thiazine-4-carbonitrile (3s)

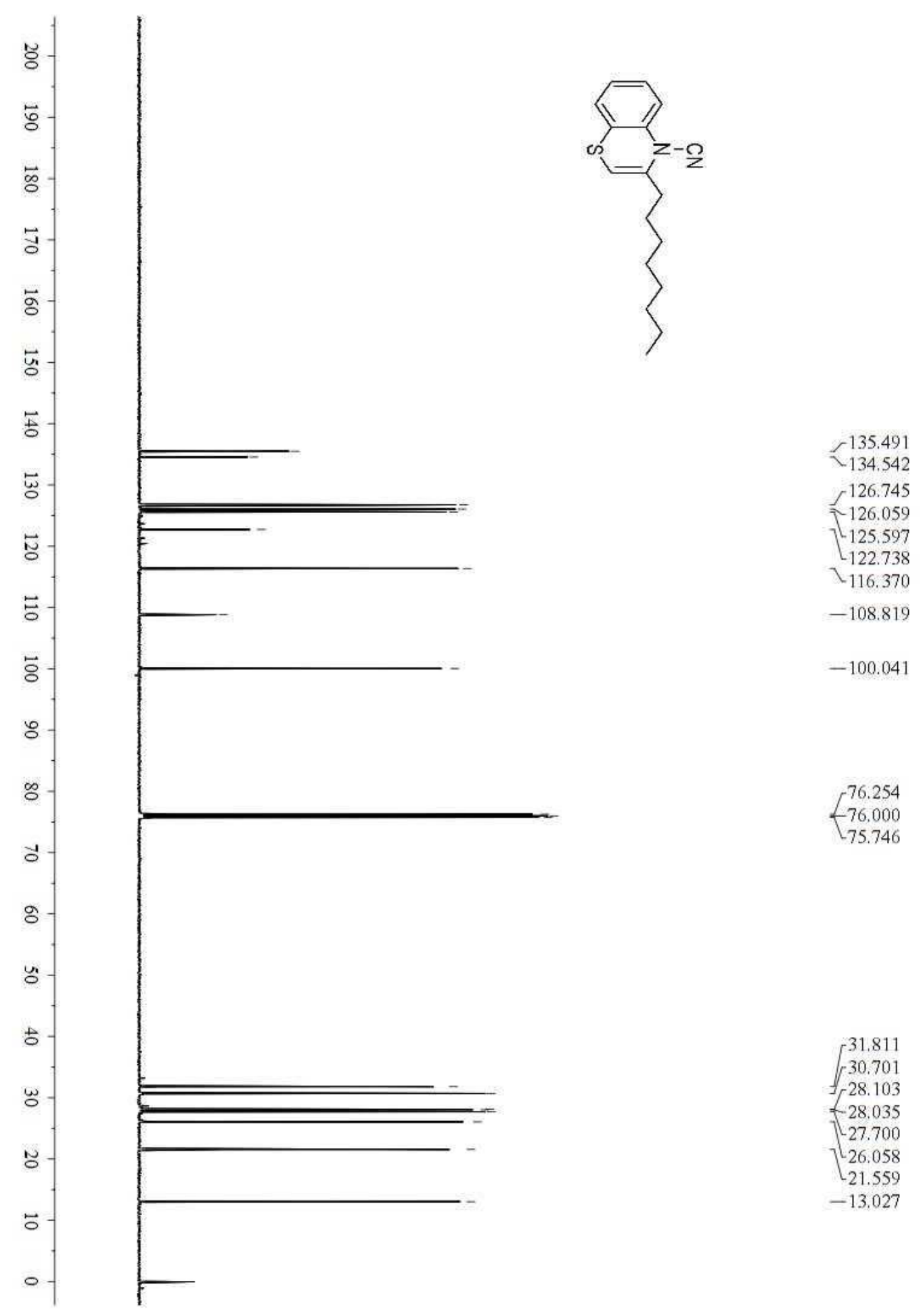


${ }^{1}$ H NMR of 7-methyl-3-phenyl-4H-benzo[b][1,4]thiazine-4-carbonitrile (3t)

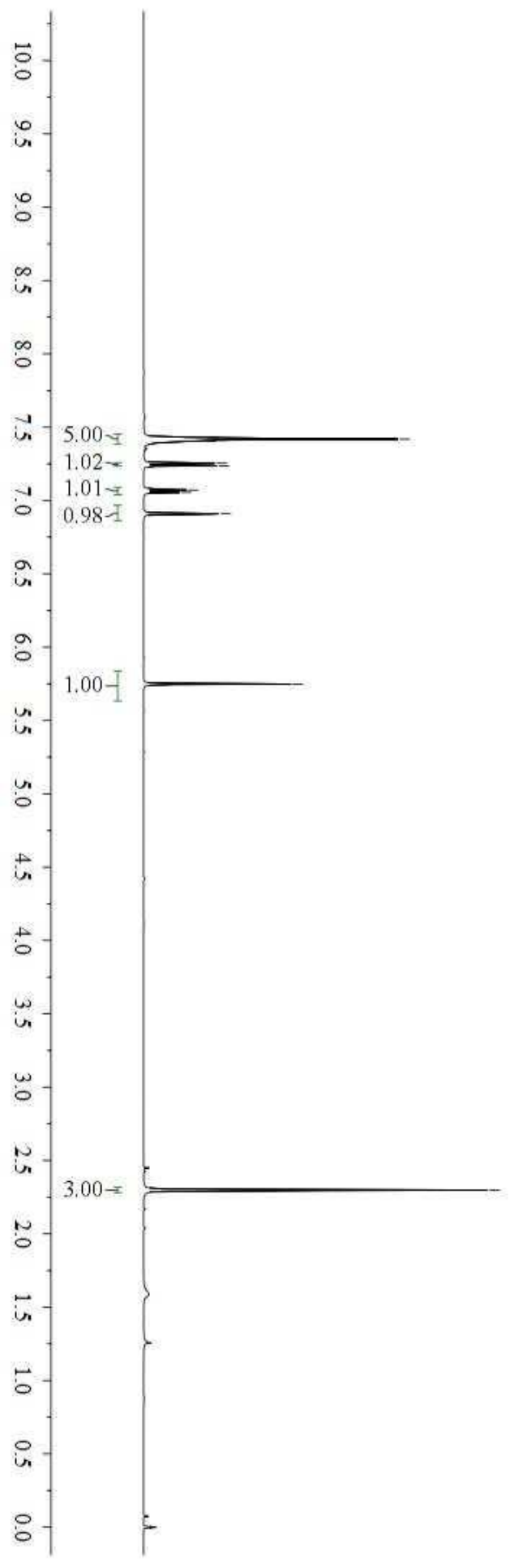


${ }^{13}$ C NMR of 7-methyl-3-phenyl-4H-benzo[b][1,4]thiazine-4-carbonitrile (3t)
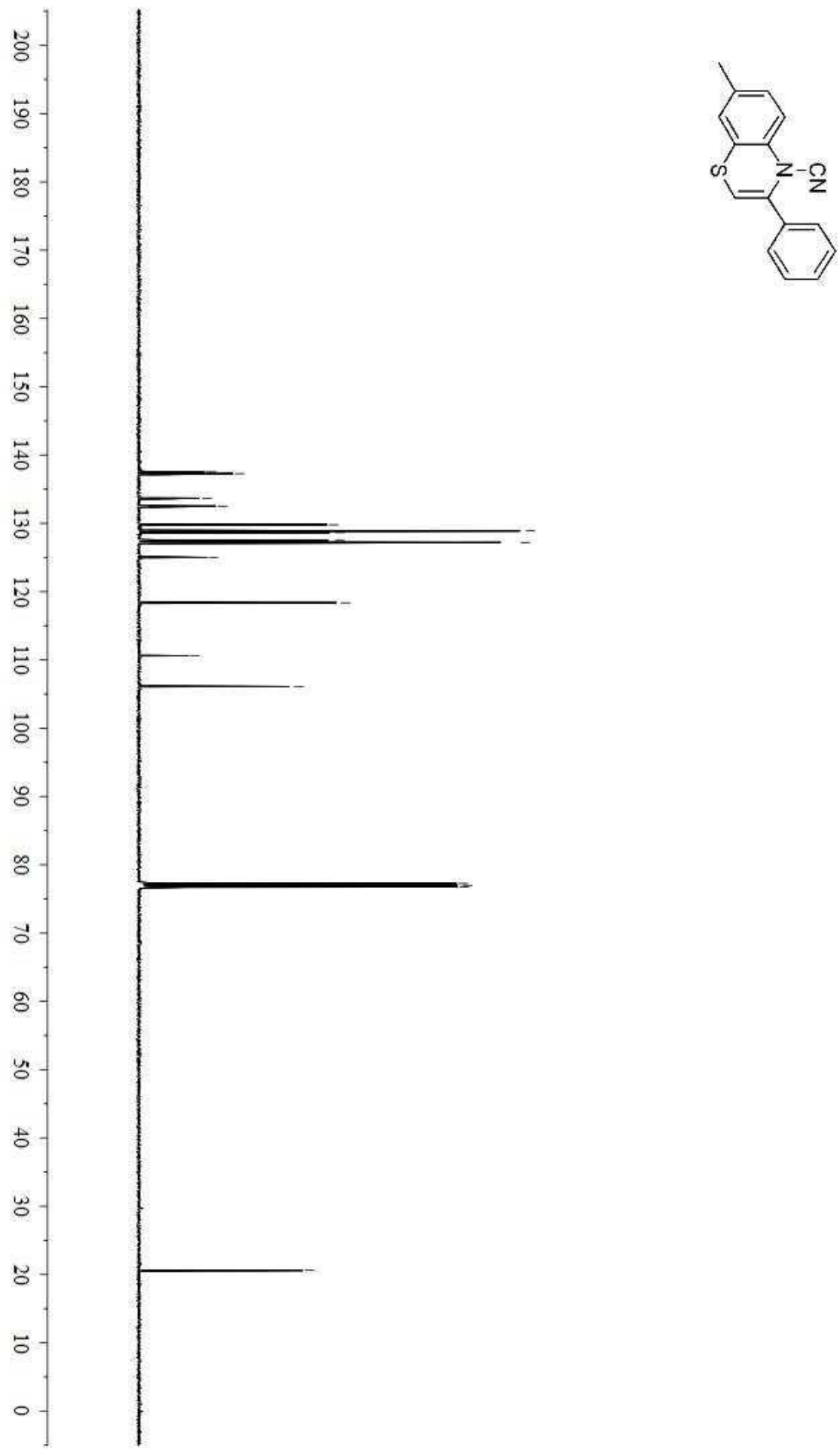

137.543

$-137.273$

133.680

$-132.517$

129.797

- 128.886

128.65

127.496

$-127.195$

$-125.001$

$-118.358$

$-110.611$

$-106.106$

77.254

$-77.000$

$-76.746$

$-20.598$ 
${ }^{1}$ H NMR of 7-methoxy-3-phenyl-4H-benzo[b][1,4]thiazine-4-carbonitrile (3u)
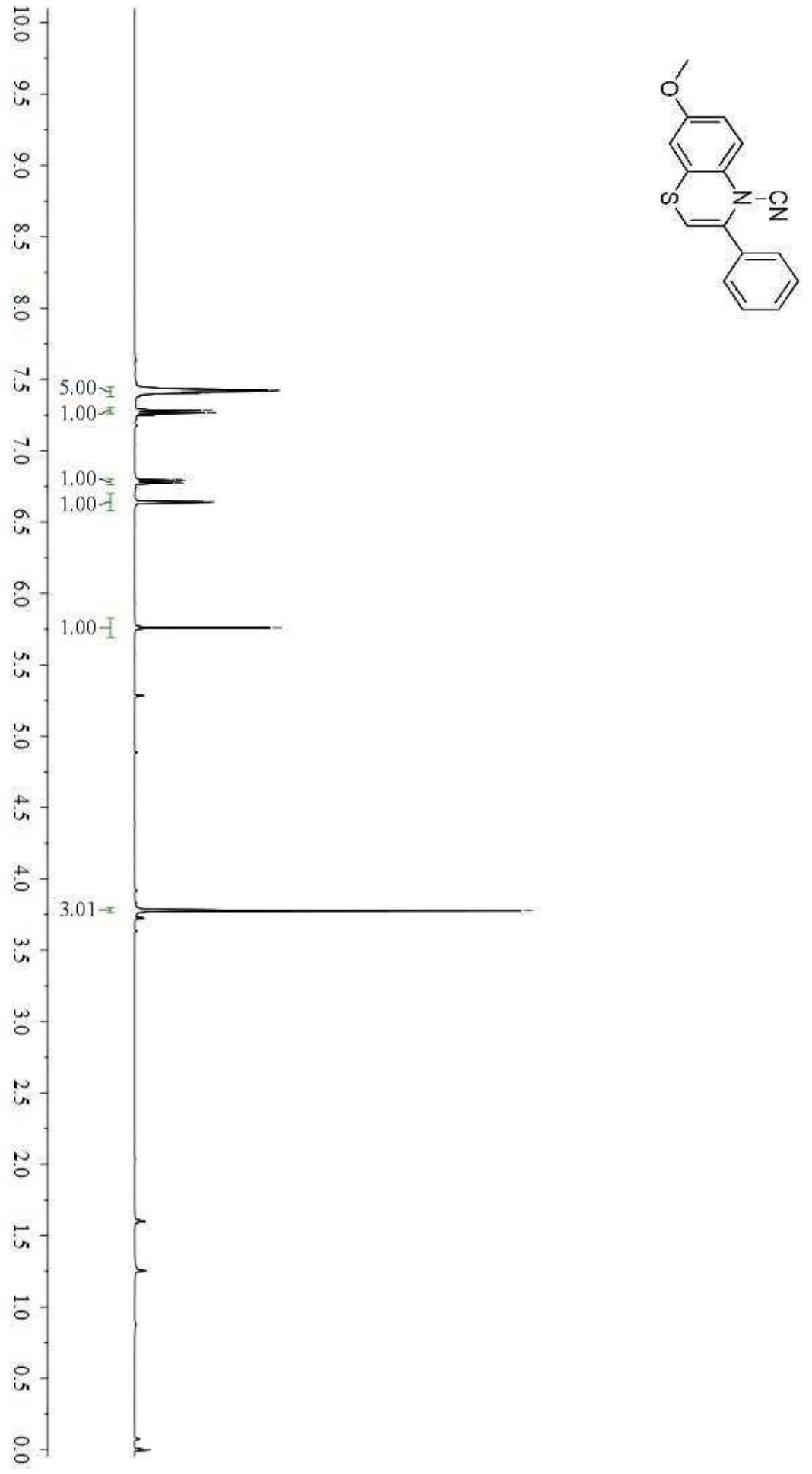
${ }^{13}$ C NMR of 7-methoxy-3-phenyl-4H-benzo[b][1,4]thiazine-4-carbonitrile (3u)

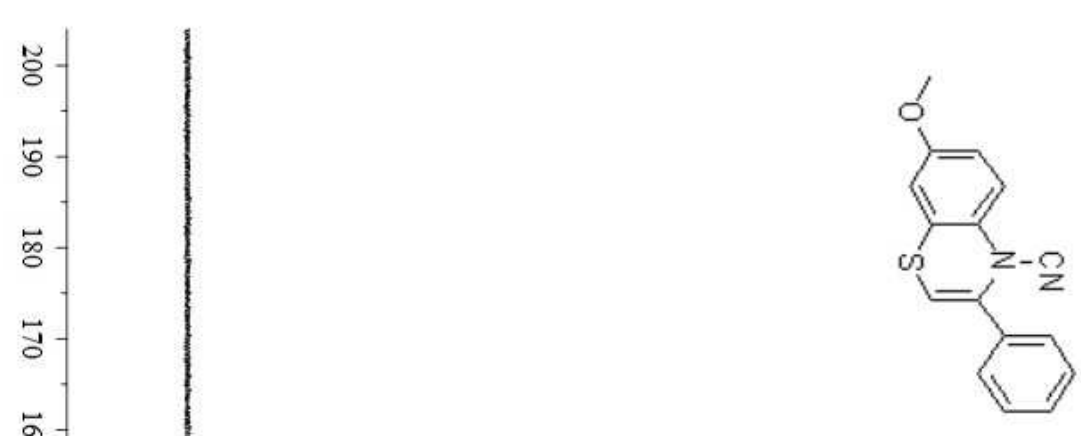

$-158.450$

$-137.856$

$-129.824$

128.885

126.897

$-119.519$

$-110.805$

$-105.746$

$-55.700$ 
${ }^{1}$ H NMR of 3-phenyl-7-(trifluoromethyl)-4H-benzo[b][1,4]thiazine-4-carbonitrile (3v)

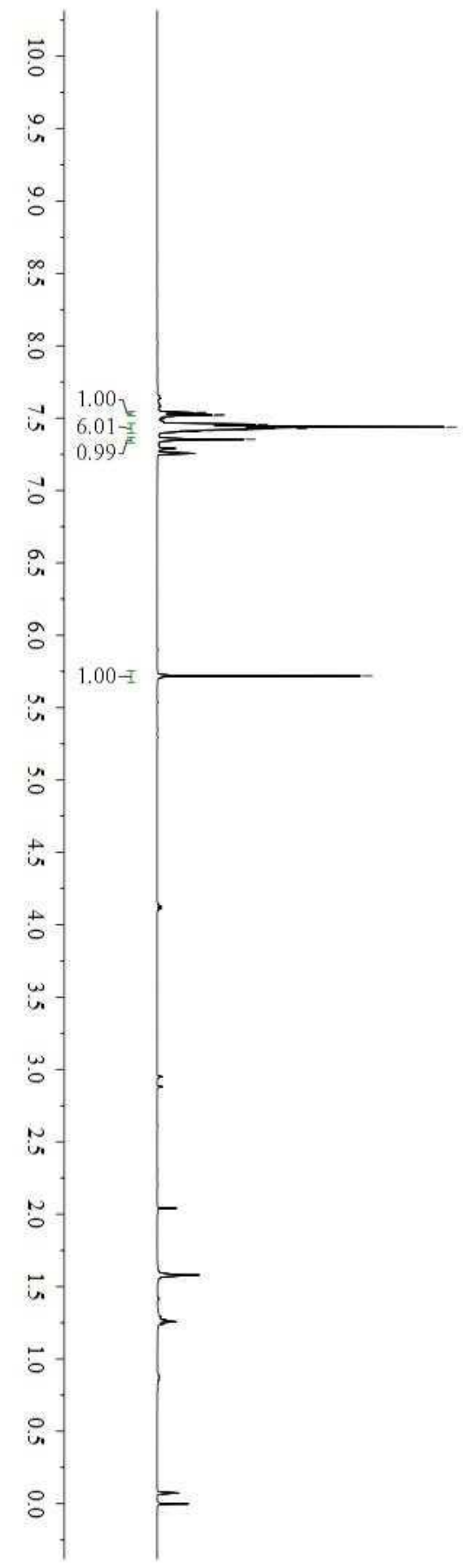




\section{${ }^{13} \mathrm{C}$ NMR of}

3-phenyl-7-(trifluoromethyl)-4H-benzo[b][1,4]thiazine-4-carbonitrile (3v)

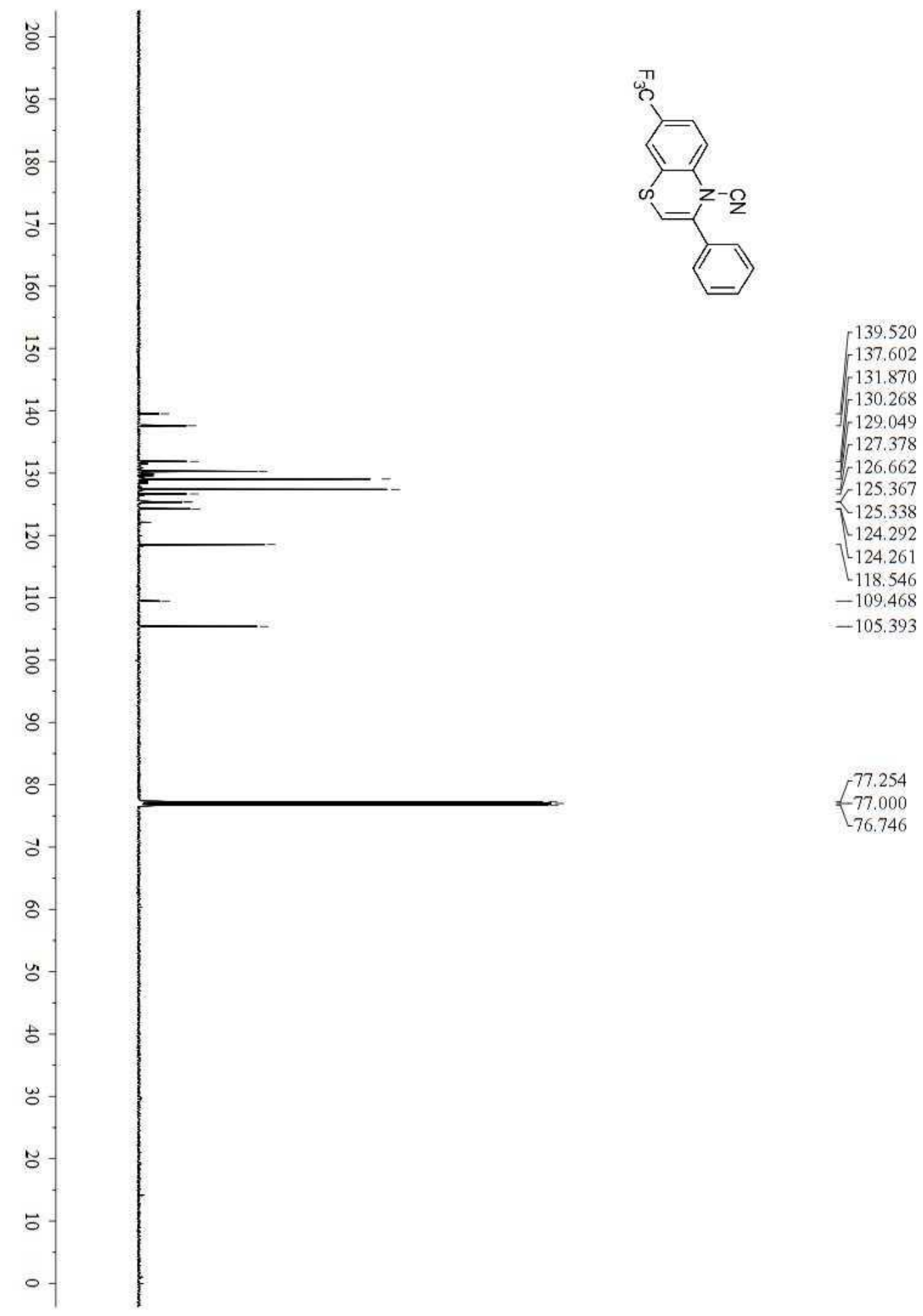


${ }^{1}$ H NMR of 7-bromo-3-phenyl-4H-benzo[b][1,4]thiazine-4-carbonitrile (3w)
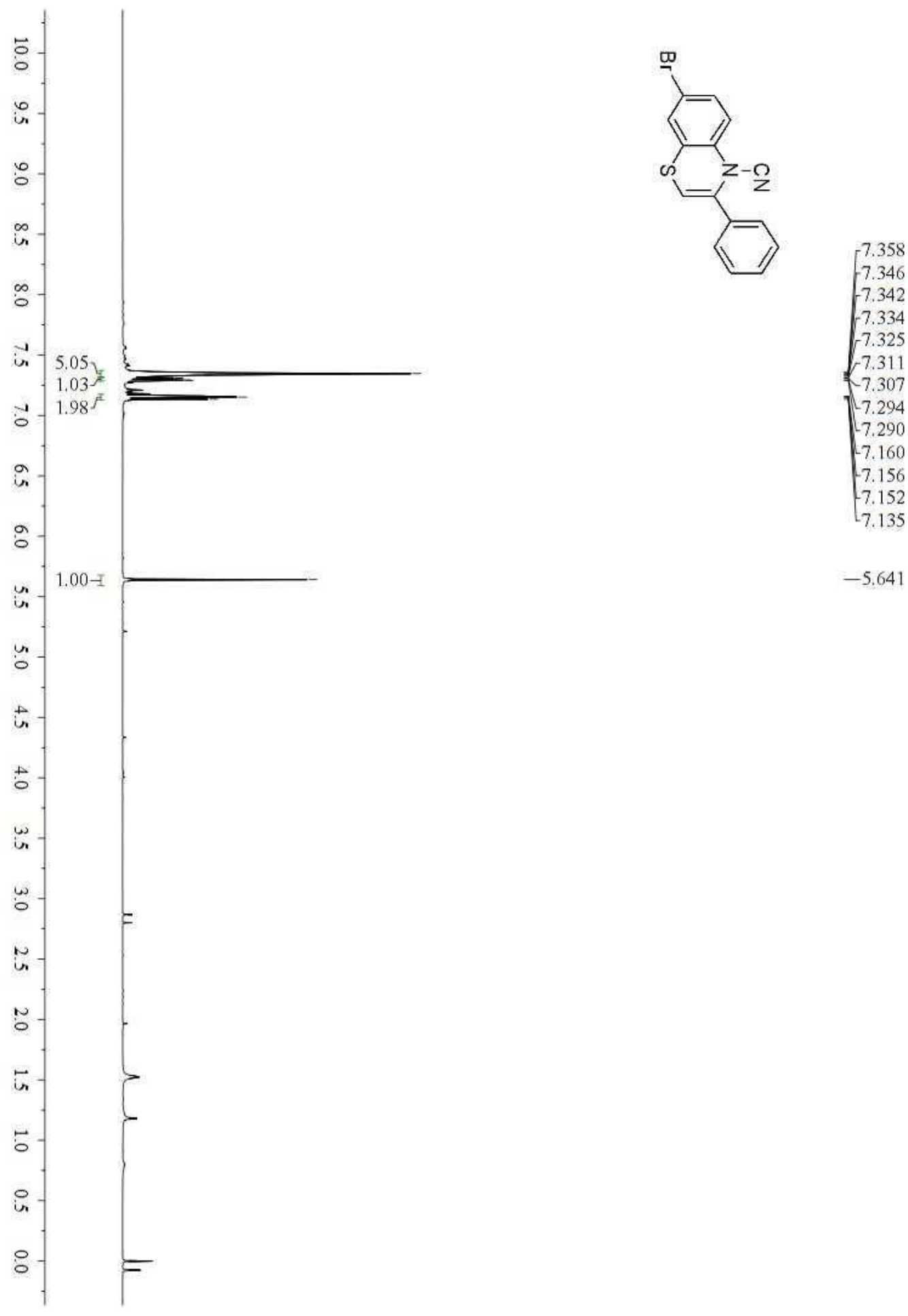
${ }^{13}$ C NMR of 7-bromo-3-phenyl-4H-benzo[b][1,4]thiazine-4-carbonitrile (3w)
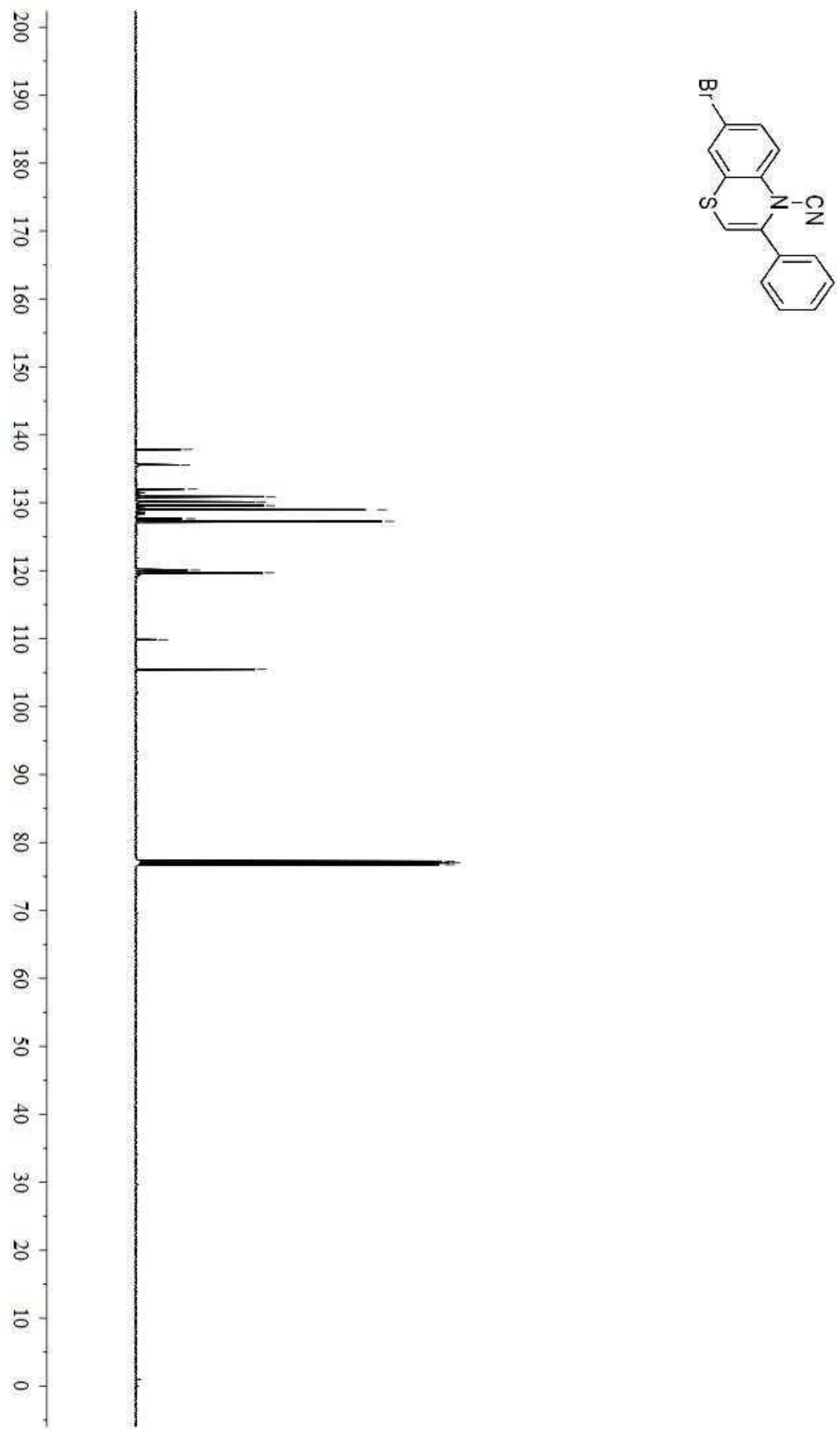

137.846

135.570

$-132.012$

130.911

$-130.114$

$-129.594$

$-128.978$

127.664

$-127.269$

$-120.079$

$-119.695$

$-109.849$

$-105.478$

77.254

$-77.000$

76.746 
${ }^{1}$ H NMR of 7-chloro-3-phenyl-4H-benzo[b][1,4]thiazine-4-carbonitrile (3x)
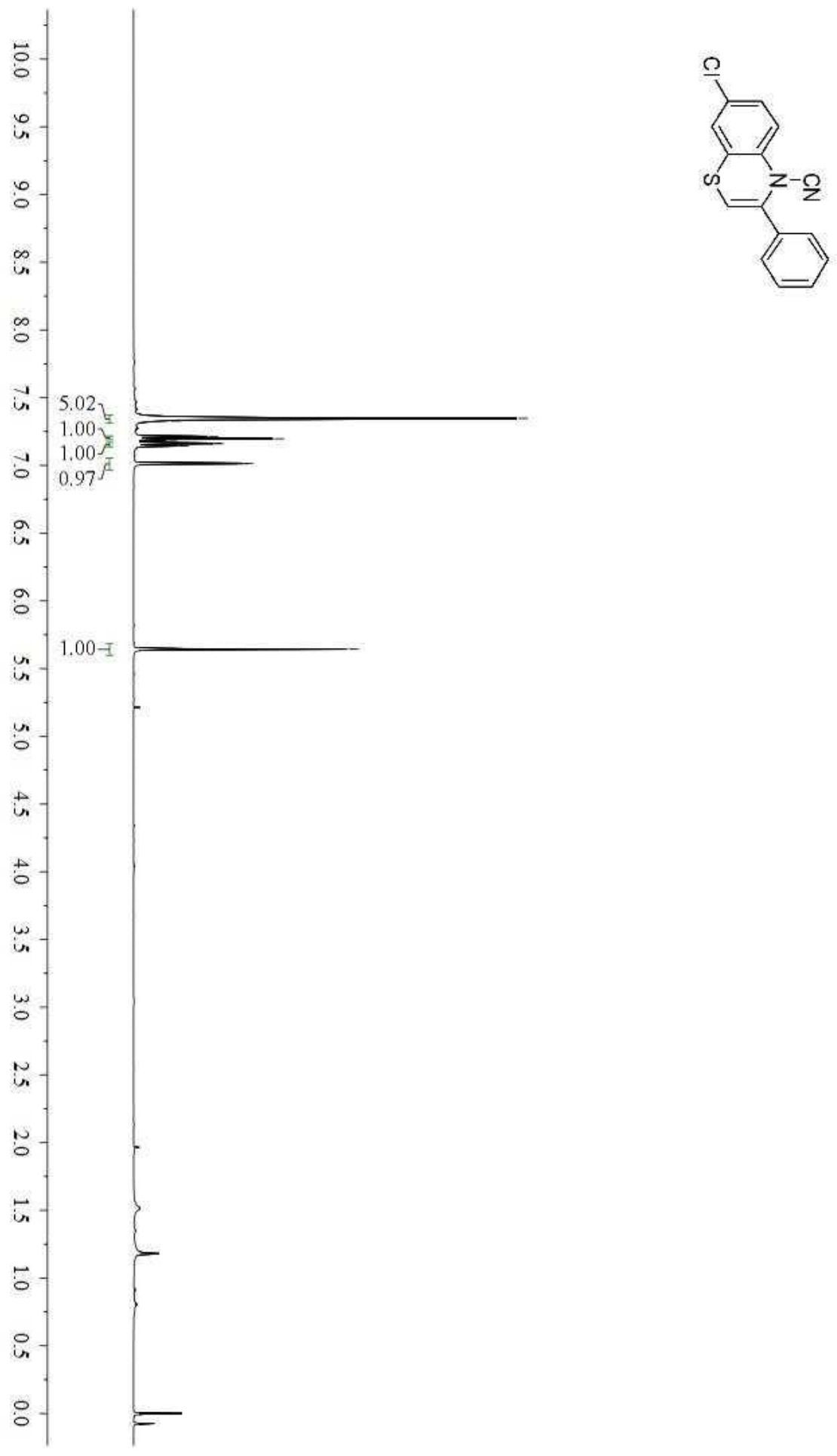
${ }^{13}$ C NMR of 7-chloro-3-phenyl-4H-benzo[b][1,4]thiazine-4-carbonitrile (3x)
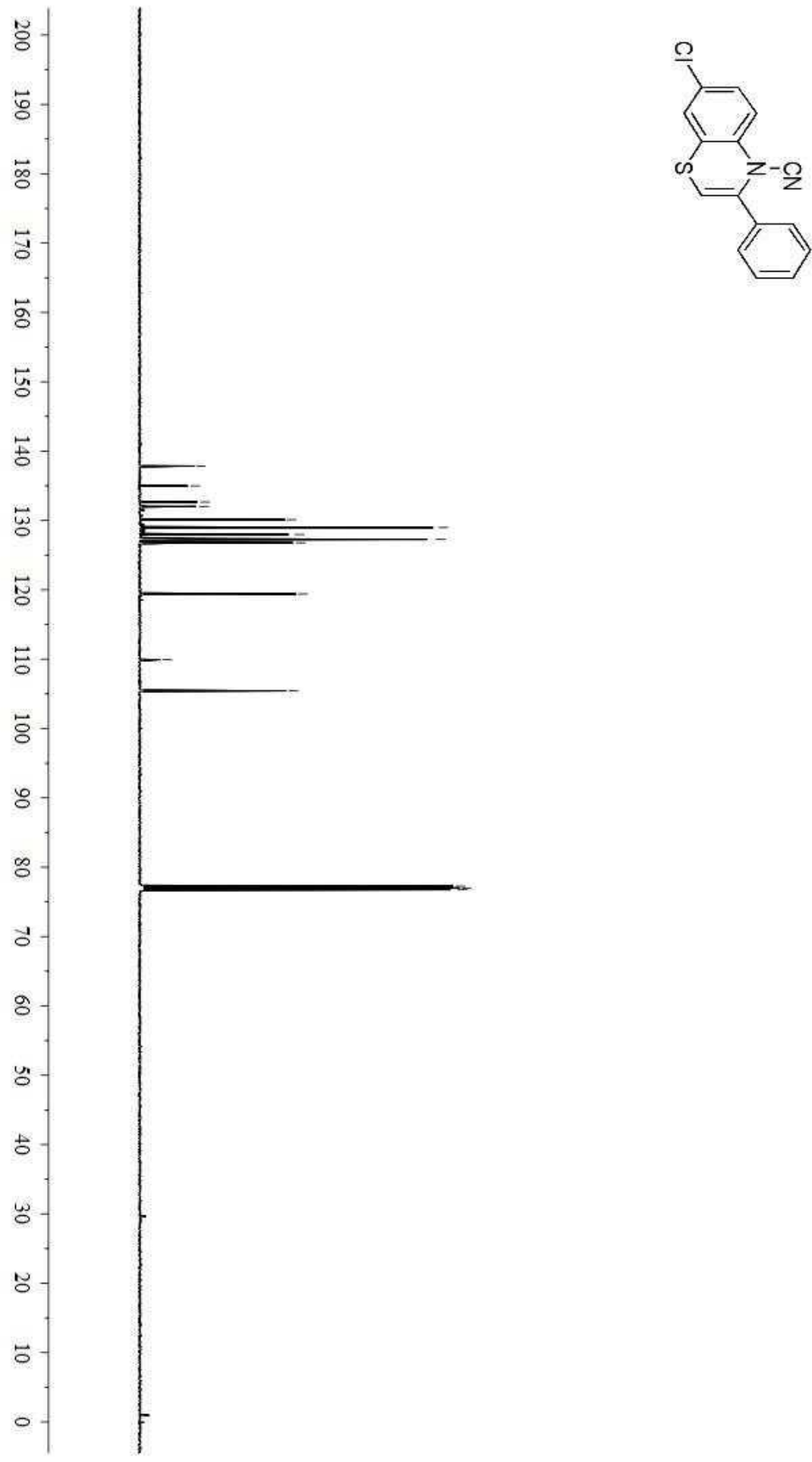


\section{${ }^{1}$ H NMR of 3-phenyl-4H-benzo[b][1,4]thiazine-4-carboxamide (4)}
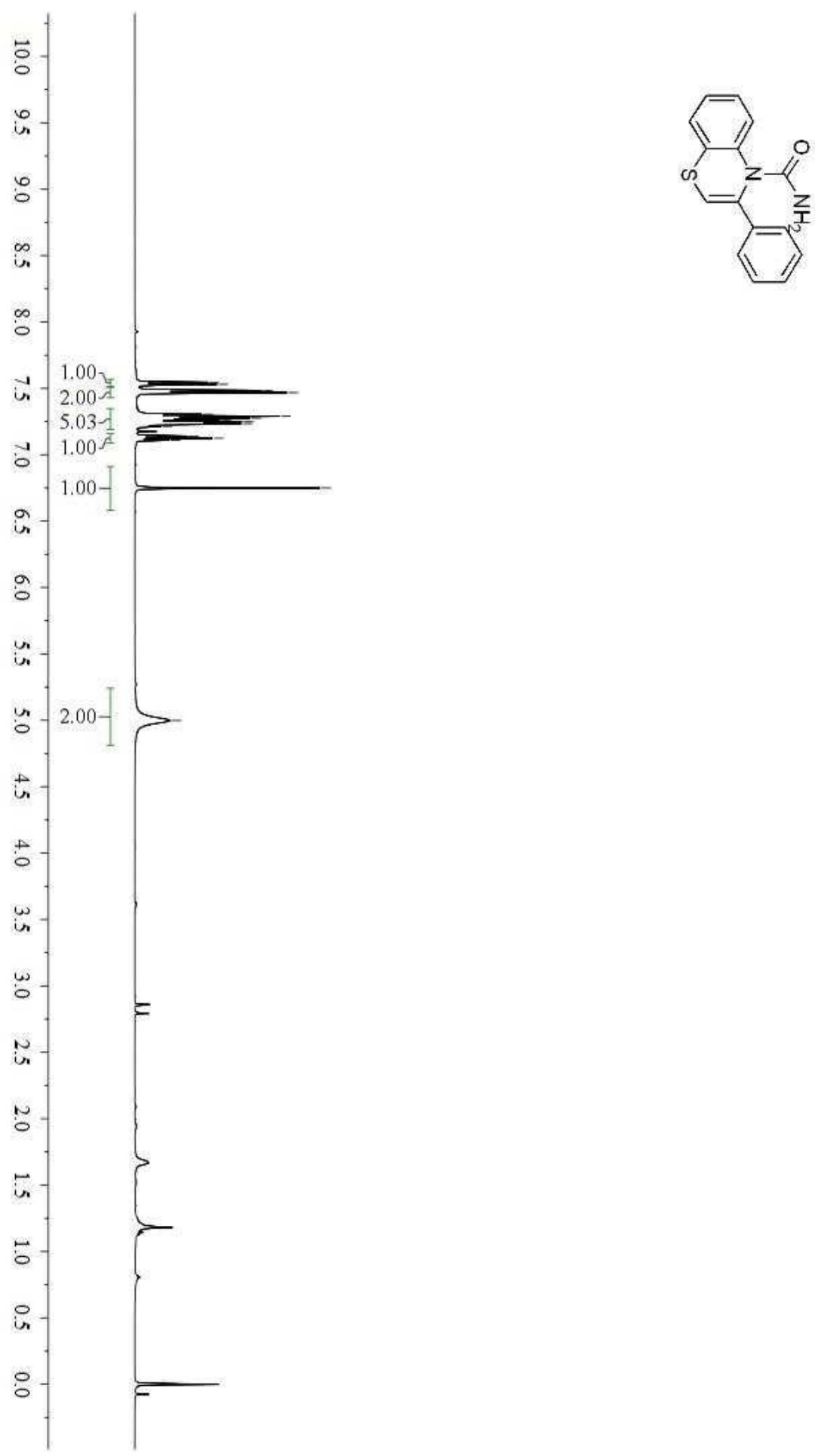

$$
\left[\begin{array}{l}
7.546 \\
7.530 \\
-7.484 \\
7.468 \\
7.305 \\
7.290 \\
7.275 \\
-7.263 \\
7.249 \\
7.235 \\
-7.229 \\
-7.214 \\
-7.140 \\
7.125 \\
7.110 \\
6.750
\end{array}\right.
$$$$
-4.999
$$ 
${ }^{13}$ C NMR of 3-phenyl-4H-benzo[b] $[1,4]$ thiazine-4-carboxamide (4)

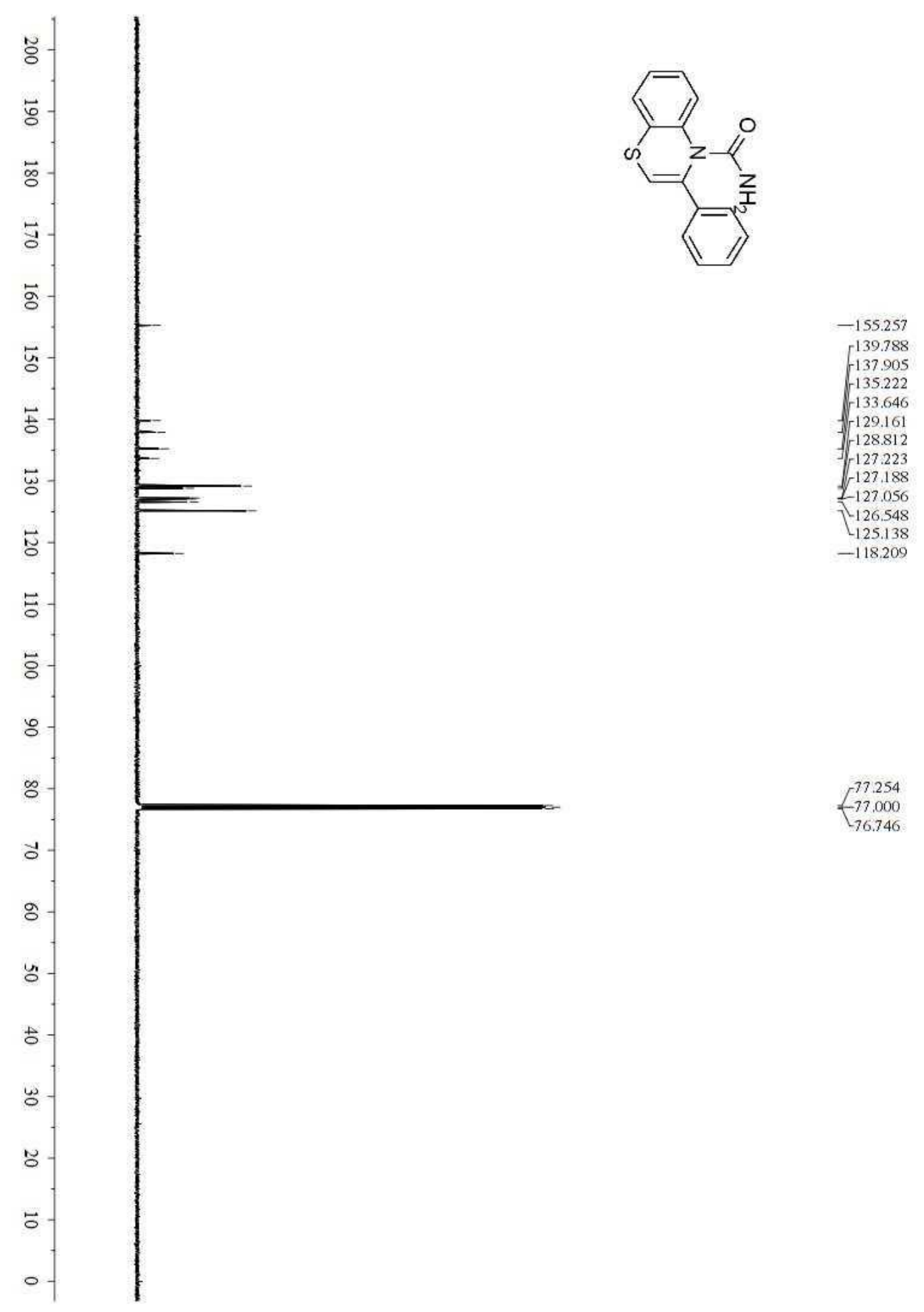


${ }^{1} \mathrm{H}$ NMR of 3-phenyl-4H-benzo[b][1,4]thiazine-4-carbonitrile 1,1-dioxide (5)
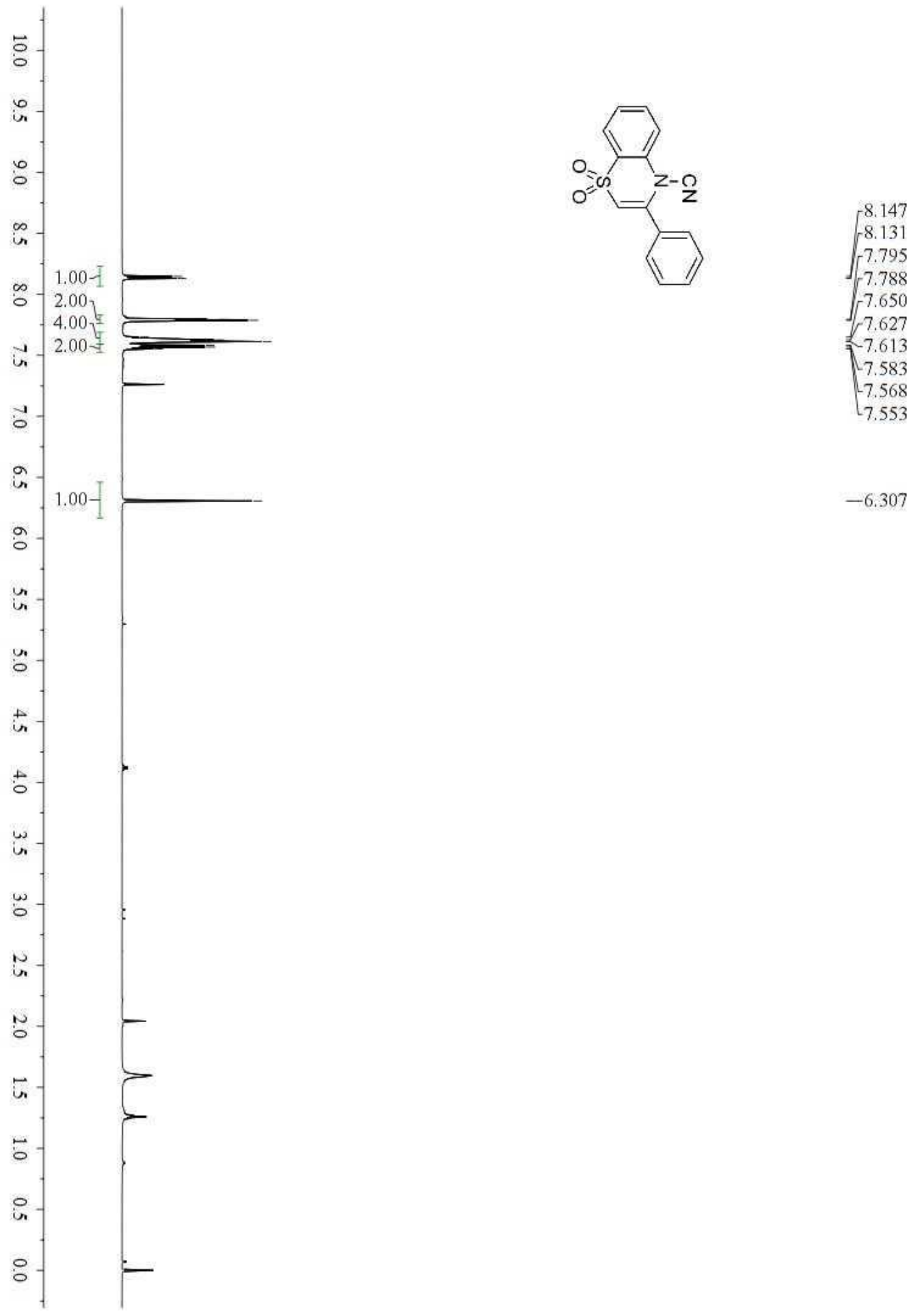

$-6.307$ 
${ }^{13}$ C NMR of 3-phenyl-4H-benzo[b][1,4]thiazine-4-carbonitrile 1,1-dioxide (5)
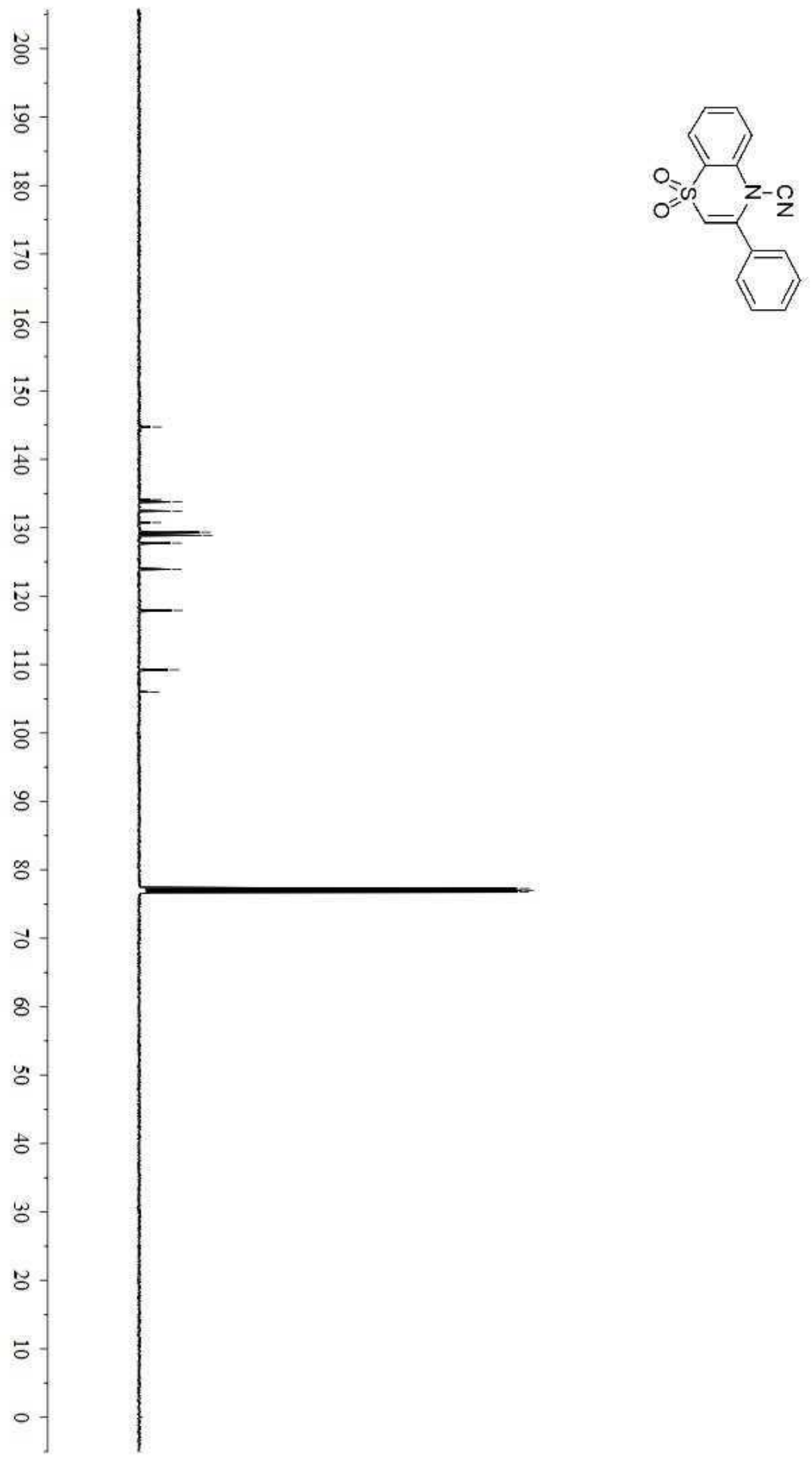
${ }^{1}$ H NMR of N-(2-chlorophenyl)cyanamide (7)

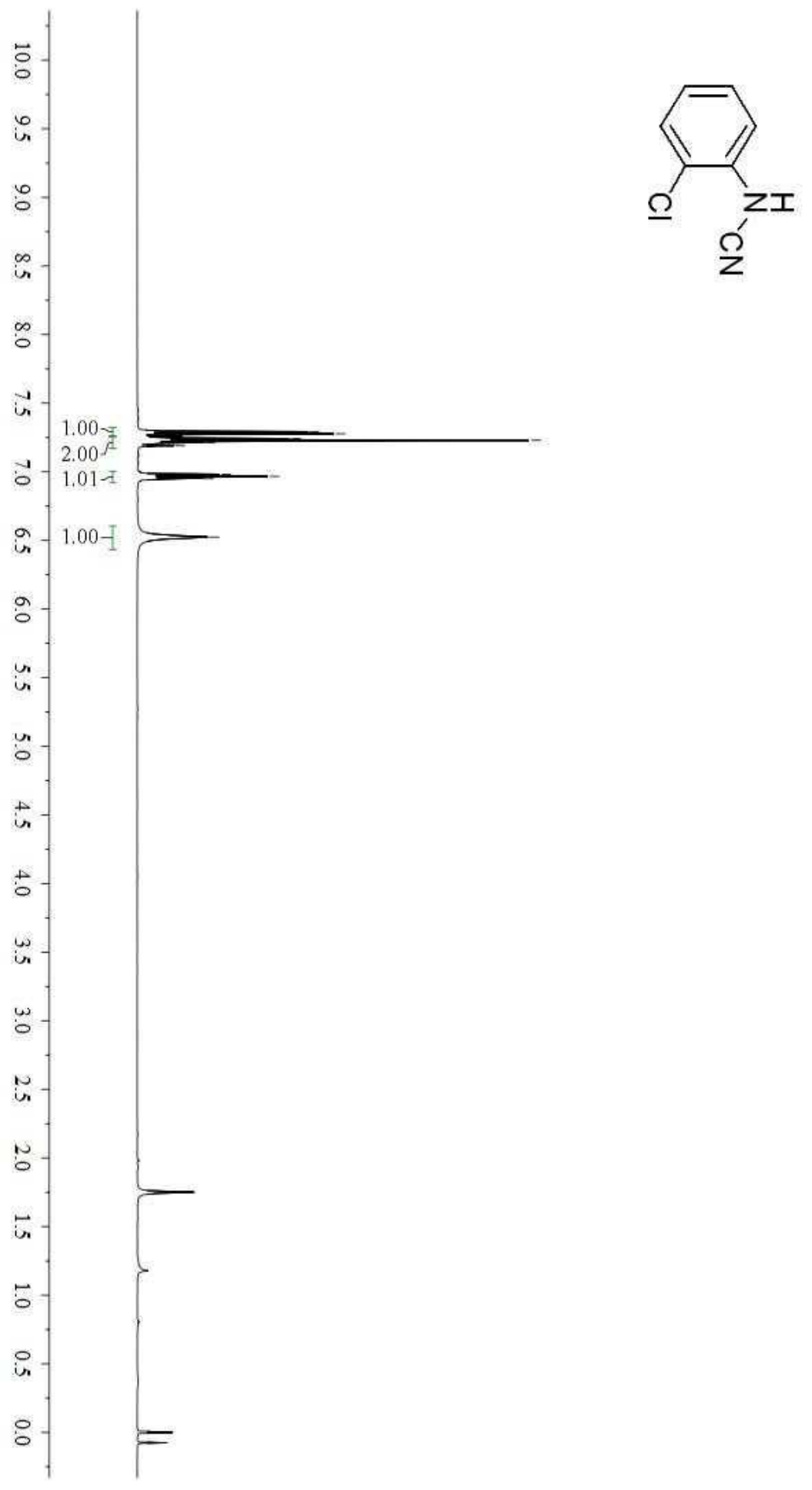


${ }^{13}$ C NMR of N-(2-chlorophenyl)cyanamide (7)
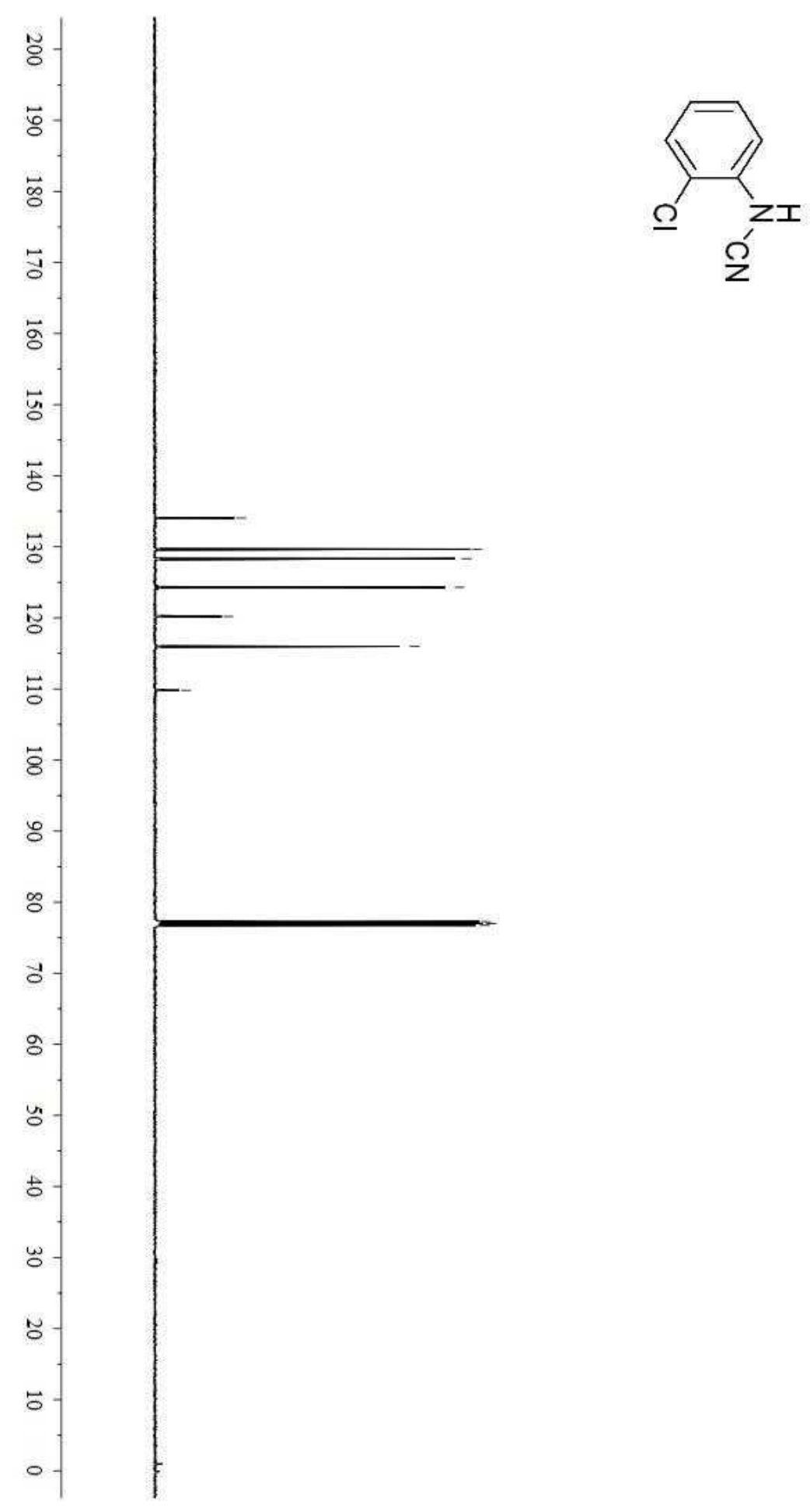

$\checkmark 134.049$

$-129.670$

128.333

$-124.298$

$\Upsilon_{120.194}$

$ح_{115,978}$

$-109.810$

77.254

$-77.000$

$-76.746$ 
${ }^{1}$ H NMR of N-phenylcyanamide (9)

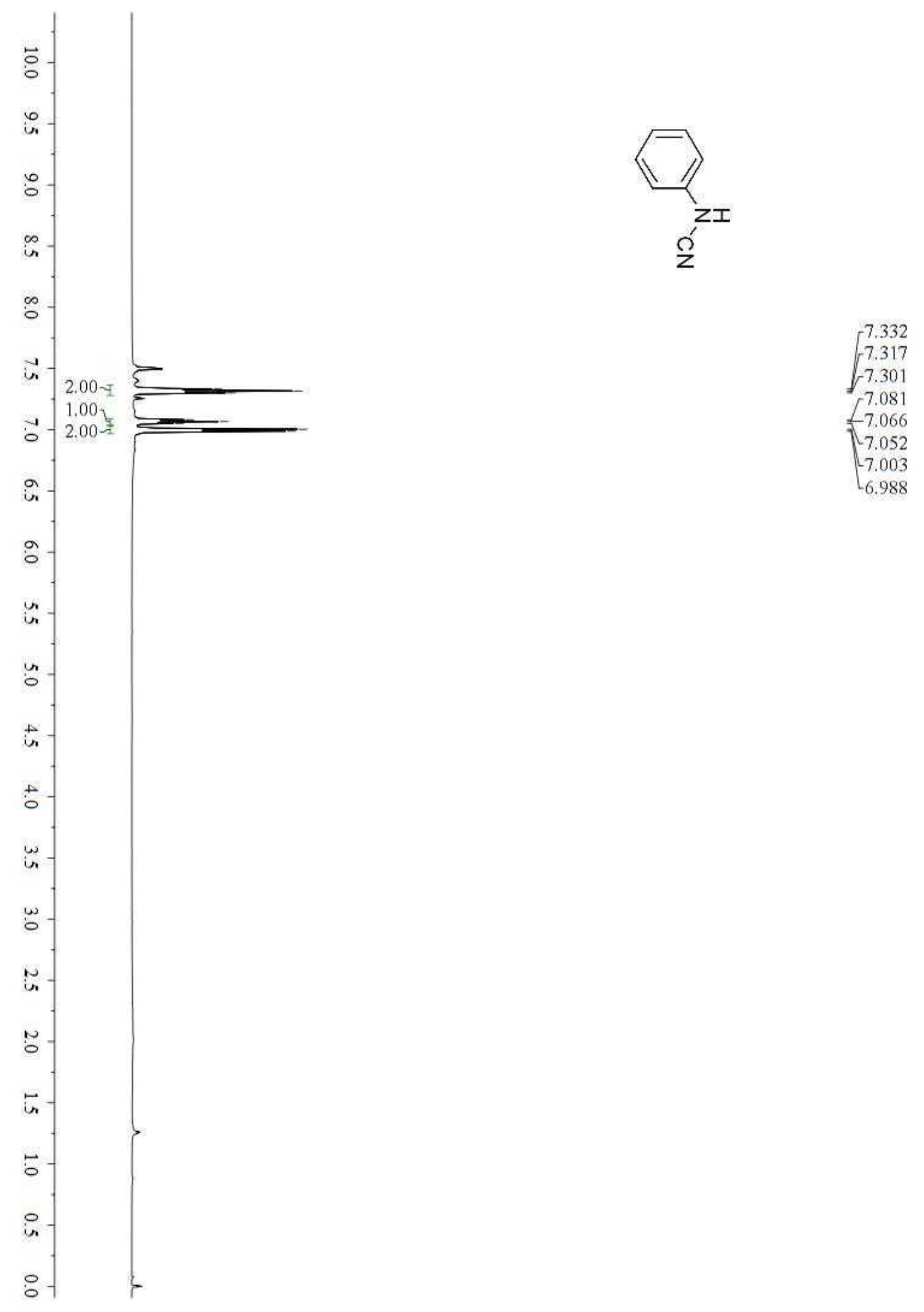




\section{${ }^{13} \mathrm{C}$ NMR of N-phenylcyanamide (9)}

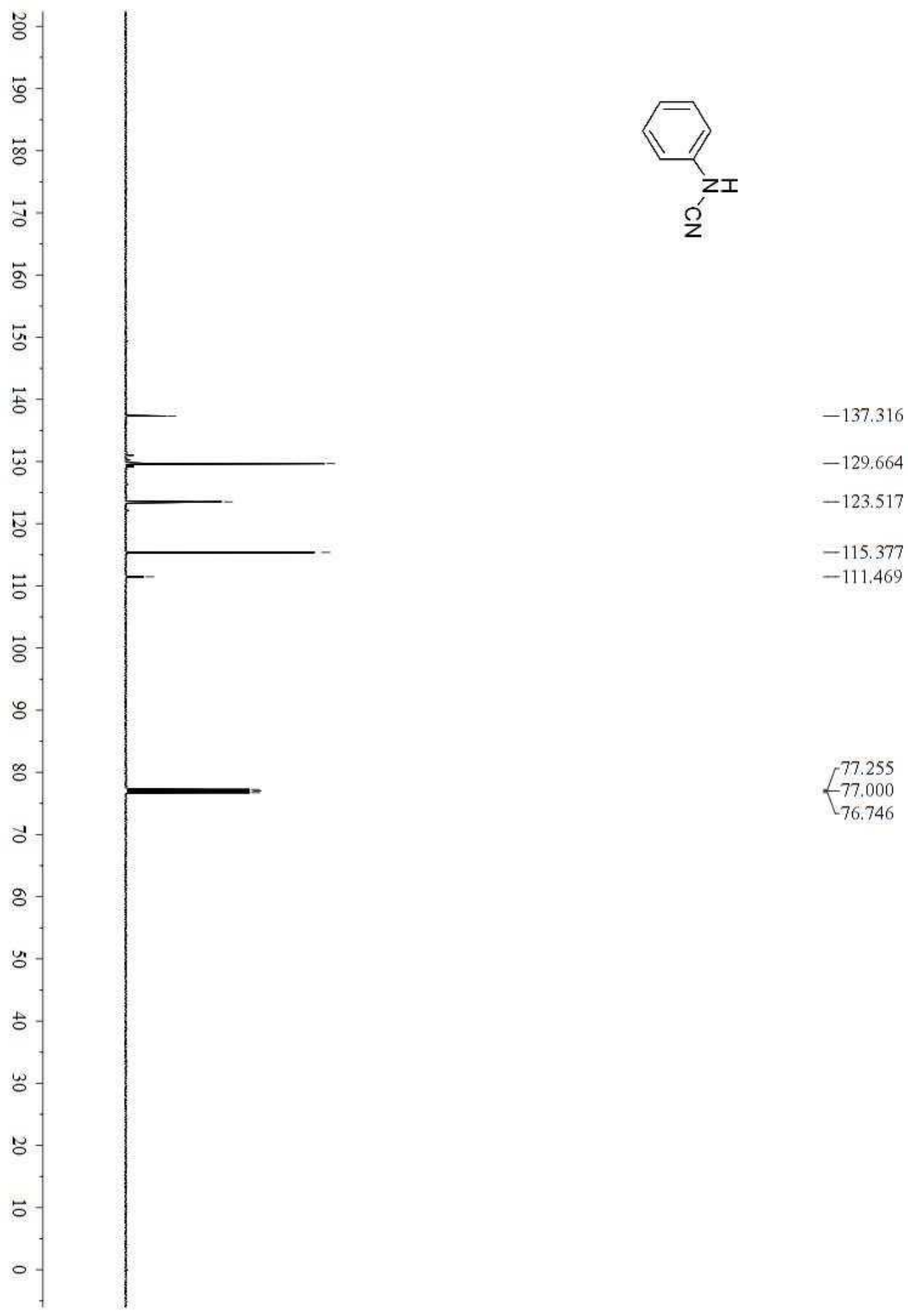

